\title{
Muster Prosa. Lineaturen der Literatur (hauptsächlich) mit Blick auf Ann Cotten
}

\author{
Glücklicher und kurzweiliger sind die Schreiber, \\ welche in ihren Werken spazieren gehen \\ und nicht eher als die Leser selber erfahren, \\ wo sie eintreffen und bleiben!
}

(Jean Paul 1963, 242)

Der Hauptunterschied bei der Arbeit an Prosa im Gegensatz zum Verfassen von Lyrik besteht für Ann Cotten darin, sich mit »Langstreckenprobleme[n]« zu beschäftigen (Cotten 2018, 130). In ihrer Poetikvorlesung Was geht (2018) befasst sie sich damit, indem sie konsequent die »Erkenntnisse von den Spaziergängen« auf Prosa projiziert (Cotten 2018, 39). Eine der Einsichten, worin Spazieren und Prosa-Schreiben vergleichbar sind und metaphorisch für das Leben in der (prosaischen) Gegenwart stehen, lautet: »Wir schreiben zu lange Werke, weil wir nicht aufhören können, weil dann die komplizierteren Probleme wieder anfangen. « ${ }^{1}$ Um die Konsequenz dieses Nicht-Aufhören-Könnens, das Probleme vermeiden will, aber auch Probleme schafft, zu begreifen und darin möglicherweise eine Theoretisierung von Prosa zu sehen, wird im Folgenden der Weg dieser langen Strecken genauer angeschaut und beobachtet, was auf diesem Weg passiert.

Um die Art des Schreib- und Spazier-Wegs von Johann Gottfried Seumes Spaziergang nach Syrakus im Jahre 1802 zu charakterisieren, benutzt Cotten ein traditionelles Bild der Theorie des Epos: »Man sieht wie der Rhythmus des langen Spaziergangs ermöglicht, die diversesten interessanten Angelegenheiten wie auf einer Perlenschnur aufgereiht zu behandeln." (Cotten 2018, 165) Bereits bei Friedrich Schlegel ist das Epos dadurch gekennzeichnet, dass es keine freien Handlungen, sondern »zufällige Begebenheit[en]« darstellt, und jede einzelne solcher Begebenheiten »das Glied einer endlosen Reihe« bildet, die tendenziell unendlich ist, da jede epische Erzählung stets nach ihrer Fortsetzung verlangt (Schlegel 1798, 106). Und noch bei Siegfried Kracauer - um nur eine weitere Position herauszugreifen, die den Fokus auf das Verhältnis

1 Cotten 2018, 165. Die Aussage ist auf Peter Handke, um den es im entsprechenden Abschnitt der Vorlesung geht, aber auch auf sich selbst bezogen.

Claudia Keller, Zürich

Ә Open Access. ( 2021 Claudia Keller, publiziert von De Gruyter. (cc) BY Dieses Werk ist lizenziert unter einer Creative Commons Namensnennung 4.0 International Lizenz. https://doi.org/10.1515/9783110731569-014 
von Epos und Episode ${ }^{2}$ und damit auf die Gestaltung solcher »Langstreckenprobleme« legt - bestehen gewisse Episodenfilme aus einer »Serie« von Einheiten, die eine relative Autonomie bewahrend wie »die Perlen einer Kette aneinandergereiht sind « (Kracauer 2005, 393). Die Affinität epischer Texte zur Prosa besteht hier unabhängig von der Frage der metrischen (Un-)Gebundenheit der Rede darin, dass beide die Geschlossenheit der Form durch eine potenziell unendliche Reihe ersetzen, die jedoch - und darauf wird es mir im Folgenden ankommen - in sich strukturiert ist.

Sich auf den Weg und in diese Strukturen hineinzubegeben, zieht eine Verlagerung des Standpunkts nach sich, sowohl konkret als auch poetologisch betrachtet - es ist kein Überblick auf eine geschlossene Form möglich, sondern es braucht eine Immersion in den Prozess: Cottens Ziel beim Spazieren ebenso wie bei Prosa ist es, die Gleichzeitigkeit der »wirren Gedankenstränge«, die in der Schreibkammer aufkommen, aufzulösen und diese »auf die Landschaft dünner aufzutragen als sonst«: »Von der überfordernden Draufsicht des Suprematismus' eines Zimmers ist man in die Ego-Shooter-Position gewechselt, und alles, was einen betrifft, kommt von selbst auf einen zu und man muss nicht wählen, welcher Sache man seine Aufmerksamkeit widmet.« (Cotten 2018, 27) Der übergeordnete Standpunkt, der in seiner gottähnlichen Gleichzeitigkeit überfordernd ist, wird durch den Überlebenskampf mit seinen unmittelbaren Reaktionen ersetzt. Ersetzt werden damit auch die Statik und Geschlossenheit im Zimmer, die so der Hinweis auf den Suprematismus - mit dem Paradigma des Bildes verknüpft sind, durch die Bewegung durch die Landschaft.

Zwar hat die Literaturwissenschaft öfters die Position bezogen, dass große Romane sich an das Paradigma des Bildes annähern, dass sie gegenüber der Lessing'schen Verzeitlichung gerade ihr Anderes - das Stauende, Stillstellende, Statuarisierende - suchen, und es ist vorgeschlagen worden, solche Bildformen als mise en abyme der Gesamtstruktur dieser Romane zu lesen. ${ }^{3}$ So zutreffend solche Lektüren im Einzelnen sind - die im Folgenden ausgeführte These geht von der Prämisse aus, dass hier weniger >Prosa « in den Blick kommt, als vielmehr die Möglichkeit von Prosa, andere Paradigmen in sich $\mathrm{zu}$ integrieren. Prosa steht dem stillgestellten Bild entgegen, sie ist Form in Bewegung und im Vollzug - be-

2 Ich beziehe mich hier auf Erkenntnisse aus der Summerschool des ZfL »Epos und Episode« von Mona Körte und Stefan Willer, 2018. 9. Oktober 2020: https://www.zfl-berlin.org/veranstal tungen-detail/items/epos-und-episode.html.

3 Sei es bspw. in den tableaux vivants in Goethes Wahlverwandtschaften oder in der Statuarisierung der Judith in der ersten Fassung von Kellers Der grüne Heinrich. Für den Grünen Heinrich hat bspw. Barbara Naumann herausgearbeitet, dass der Roman »seine Abhängigkeit vom Bildparadigma« auf verschiedene Weise ausstelle (Naumann 2009, 178). 
reits so lässt sich die vielfach nachgewiesene Affinität zwischen Prosa und Spaziergang begreifen (Albes 1999; Hahn et al. 2017). Dabei ist es aber nicht so, dass der Literatur und insbesondere der Prosa durch ihre Verknüpfung mit der flüchtigen, formlosen Zeit alle Möglichkeiten offenstehen würden. Nur weil Prosa immer voranschreitet, heißt das nicht, dass sie auch alles hinter sich lassen kann - genau dies ist mit dem Hinweis auf die »Langstreckenprobleme« gemeint. Prosa als sermo pedestris, als die zu Fuß gehende Rede, wie Horaz sie bezeichnet hat, hinterlässt Spuren, sie bildet und in ihr vollzieht sich Form, sie entwickelt Mechanismen der Selbstbindung, in denen sie sich materialisiert. ${ }^{4}$ Mir geht es weniger um die Diskussion >Prosa vs. >Form $<{ }^{5}$ als dass ich auf die Ausbildung von Strukturen fokussiere und so nach einer spezifischen Prosa-Form frage, die ich als >Muster Prosa bezeichne. Um die ıspezifische Dichte $\mathrm{zu}$ beschreiben, die Prosa laut den Prämissen des Basler SNF-Projekts "Theorie der Prosa « herstellt, ${ }^{6}$ verfolge ich die Linien, die bei der Bewegung dieser Texte entstehen und untersuche die Muster, die daraus hervorgehen. Ich stelle die ornamentalen Strukturen und also die Vor-Bildlichkeit komplexer literarischer Prosatexte ins Zentrum und frage mit einem bildwissenschaftlichen Fokus danach, wie sich Prosa zur Anschauung und zur Darstellung bringt. ${ }^{7}$

Ein Muster ist Bewegung und Strukturierung zugleich, es besteht im Wechsel von Variation und Wiederholung. Wenn Cotten beim Verweis auf Seumes Spaziergang vom >Rhythmus` spricht, dann benennt sie explizit ein solches Muster, das auch von der literaturwissenschaftlichen Theoriebildung bereits vielfach als Kennzeichen für Prosa herangezogen wurde. ${ }^{8}$ Helmut Hühn hat jüngst mit Blick

4 Für den Hinweis auf Horaz: Lüdemann 2019, 322; für Prosa als Verfahren der Selbstbindung ebd., 316 und 324 sowie Mülder-Bach 2019a, 10 f. sowie 2019b, bes. $239 \mathrm{f}$.

5 >Prosa als etwas, das >vor und nach der Form` liegt, hat Ralf Simon theoretisch ausformuliert und mit Blick bspw. auf Jean Paul, James Joyce oder Arno Schmidt gezeigt. Er setzt damit Prosa (= Nichtform) von Erzählung (= Form) ab (Simon 2018, bes. 416 sowie Simon 2016). Für (historische) Vorüberlegungen zum aktuellen Projekt: Simon 2013. Zur (teilweise kritischen) Auseinandersetzung mit diesen Thesen: Campe 2016, bes. 46, sowie die Sammelbände mit insgesamt anders gelagerten Ansätzen: Mülder-Bach et al. 2019 sowie Efimova und Gamper 2020, dort auch weitere Hinweise auf die Forschung.

6 Zum Projekt: 9. Oktober 2020: http://p3.snf.ch/Project-173039. Zur spezifischen >Dichte vgl. Simon 2018, bes. 424 sowie bereits 2013, bes. 290, wo die Frage nach der Dichte als »Kernproblem der Literaturwissenschaft« formuliert und eingefordert wird, sie von einem anderen Standpunkt als demjenigen der Form zu bedenken.

7 Simon $(2018,419)$ hebt die zentrale Funktion des Darstellungsbegriffs für Prosa hervor, dabei geht es auch um eine Verschiebung von der Poetik zur Ästhetik (ebd., 428). Mit der Aufmerksamkeit auf die Linie und das Muster schneide ich das von Simon (2013, 291) formulierte Desiderat, einen Prosabegriff für die bildende Kunst zu denken, zumindest an.

8 Simon 2018, 415, Mülder-Bach 2019a, 2 sowie besonders: Gamper 2021. 
auf die frühgriechische Lyrik (und die Transformationen in der Philosophie) eine Perspektive auf das Rhythmus-Konzept herausgearbeitet, die das problematische Verhältnis von Form und Zeit fokussiert. Rhythmus ist »Denken der bewegten Form und der geformten Bewegung" (Hühn 2020, 74). Und: „Im Rhythmus gewinnt Zeit eine Form (jenseits der Abfolge), wird sie wahrnehmbar und erkennbar.« (Hühn 2020, 76) Die Muster, die ich im Folgenden betrachte, stellen ebenfalls einen solchen Rhythmus dar, wenn auch - mal grafisch, mal metaphorisch - auf die visuelle Ebene übersetzt. Zeit ist so gesehen nicht das Gegenteil von Form, sondern in der Zeit passiert Form. Dies ist auch der für eine Theorie von Prosa interessante Gedanke bei Cotten: In ihren Texten stehen alle möglichen Formen musikalischer, sprachlicher und visueller Rhythmen für eine Bewegung und Dynamisierung von Literatur, die auch für eine Generation einsteht, die sich dem Erwachsenwerden entziehen will; gleichzeitig führen diese Verfahren zur Erkenntnis, dass auch hier die Möglichkeiten begrenzt sind: Prosa erscheint zunächst - sowohl poetologisch als auch auf das Leben bezogen - als unendliche Möglichkeit, immer weitere Schichten aufzutürmen, aber vor allem bedeutet Prosa, sich einzugestehen, dass diese Schichten real werden, dass sie sich >materialisieren`, ablagern, dass sie eine Folge und eine Wirkung haben, Formen annehmen, die manchmal Verkrustungen bilden und die durchaus chimärisch sind.

\section{Faltung in sich selbst: Die Verstrickung in selbstproduzierte Muster auf den Wegen der Prosa}

Wird der Suprematismus durch das Videogame ersetzt, geschieht nicht nur eine Ablösung des Bildparadigmas der Gleichzeitigkeit durch die Prozessualität des Vollzugs, die sich im dünnen Auftragen von Linien auf die Landschaft zeigt. Die praxeologische Wendung, in der die wirren Gedanken auf die körperliche Erfahrung verschoben werden, erstreckt sich auch darauf, dass eine Auseinandersetzung mit der >prosaischen Welt‘ und mit den Gegenständen gesucht wird. Der Suprematismus, der sich bereits dem Namen nach mit dem >Höchsten schäftigt, bringt gemäß Kasimir Malewitsch in seinem Kommentar zum Schwarzen Quadrat die >reine`, also gegenstandslose »Empfindung« zur Darstellung (Malewitsch 1927, 65). Er dient hier als Kontrast zu einem Realismus, der Schluss macht mit reiner Selbstbespiegelung und die Konfrontation einfordert, mit dem, was passiert: 


\begin{abstract}
Und es passiert zum Glück immer noch was, bzw. man geht daran vorbei, also durch die Wahrnehmung der Momente hindurch. Als wäre der Spaziergang ein Ritterroman, eine abenteuerliche Reiterei durchs Bewusstsein. Kaum ist ein Monster besiegt, sei es ein schlechtes Gewissen, eine Erynnie, eine Chimäre, ein Schatten, eine Tagträumerei, sprießt schon das nächste Ding, als Neuigkeit, aus den neuen Ereignissen. Und ist das nicht wie der Cursor im Schreibprogramm? Nimmt man die Mittagspause als Zäsur, ist sogar ein sogenannter »Doppelter Cursus« möglich. Man lässt die Drachen, wie die Autornie eines Ritterromans, nach und nach herauskommen, besiegt sie, und kehrt als Held seiner Müdigkeit heim. ${ }^{9}$
\end{abstract}

Das zeitgenössische Videogame ist mit dem mittelalterlichen Ritter- und Abenteuerroman vergleichbar, beide stehen stellvertretend für die »Langstreckenprobleme« beim Verfassen von Prosa: Die Aufmerksamkeit ist nicht gebündelt, sondern zerstreut, immer kommt etwas dazwischen, Monster nach Monster muss besiegt werden, bis zur Erschöpfung. Durch den Vergleich des Spaziergangs mit der »abenteuerliche[n] Reiterei durchs Bewusstsein« wird aber die soeben gezogene Trennung von Natur und Vorstellung, von Empirismus und Idealismus gleich wieder kassiert. Ob es tatsächliche Monster oder Monster der eigenen Vorstellung sind, kommt aufs Gleiche heraus - man lässt sie herauskommen, versucht sie zu besiegen, nur um als Held der eigenen, selbstproduzierten Müdigkeit heimzukehren. Der >doppelte Cursus bei Ann Cotten besteht nicht darin, dass der Held an den Gefahren, die er in der Welt vorfindet, wächst und eine Korrektur vornehmen kann, sondern er besteht in der Rekursivität des Hin und Her, die eine Faltung in sich selbst bedeutet: Man produziert fortlaufend selbst, was es dann zu überwinden gilt.

Was bedeutet der Gang dieser Prosa für die Reflexion eines Lebens in der Gegenwart? Worin besteht die Metaphorisierung der "Langstreckenprobleme« von Prosa für die Wahrnehmung der (post-)modernen Gesellschaft? Wenn, wie Inka Mülder-Bach (2019b) anhand von Goethes Unterhaltungen deutscher Ausgewanderten gezeigt hat, anhand der >ungebundenen« Prosa auch die >Bindungslosigkeit der modernen Welt reflektiert wird, die sich von ihren Normen, aber auch ihren sozialen Strukturen gelöst hat, dann besteht Cottens kritische Reflexion auf das vermeintliche anything goes (post-)moderner Gesellschaften darin, dass, ehe man sich's versieht und gerade dort, wo man es nicht vermeint, eine Bindung als Selbstbindung geschieht, die jedoch keine selbstbewusste Entsagung, sondern ein Kollateralschaden ist. In Cottens Gegenwart besteht die Konsequenz der Hegel'schen `Prosa der Welt‘ bzw. der >Prosa der Verhältnisse nicht nur darin, dass das Individuum von den normativen bürgerlichen Ord-

9 Cotten 2018, 40. Bei der Formulierung `Autornieく handelt es sich um sog. `polnisches Gendering`, vgl. dazu weiter unten. 
nungsstrukturen und ihren entsprechenden Institutionen abhängig ist (zu Hegel: Mülder-Bach 2019a, 9f.), sondern die Prosaisierung hat auf das Innere des Subjekts übergegriffen. Es hat die Machtverhältnisse vollkommen internalisiert - Formung der Welt wie auch der Prosa vollzieht sich als Verstrickung in selbstproduzierte Muster.

Mit Verweis auf das Nicht-aufhören-Können des Schreibens bzw. Spazierens macht Cotten auch darauf aufmerksam, dass das Konzept des Eskapismus nicht funktioniert - bei der Flucht vor den Gedanken beim Spazieren oder Schreiben handelt man sich dieselben Probleme wieder ein, die man hinter sich zu lassen vermeint. Der auf diesen Gedanken folgende Abschnitt ihrer Vorlesung verweist denn auch auf die Tatsache, dass «man sich beim Gehen - wie beim Abenteurern und Leben, letztlich bei jeder dauerhaft aktiven Tätigkeit - in sich selbst ein[legt] wie ein Gemüse. Die Seele vergurkt sozusagen." (Cotten 2018, 40) Durch die Wiederholung wird das Aktive dauerhaft und konserviert sich selbst. In der Analogisierung von Leben und Schreiben, wie sie hier von Cotten etabliert wird, bedeutet dies wiederum: >Prosa` passiert, wenn Bewegung und Gestaltung in Muster und Strukturbildung umschlagen.

Als Problematik ergibt sich so, dass die herkömmlichen Vorstellungen von vollendeter Form für beides, das gelebte Leben und den Text, nicht realisiert werden können. Wenn die Seele svergurktı, wird sie zu einem hybriden Mischwesen, das für Cotten zu einer zentralen poetologischen Denkfigur avanciert: Wenn Schriftsteller ${ }^{\star}$ innen sich in ihrem Besonderheitsdrang auf neue Wege begeben, dann gehen daraus nicht unbedingte neue Formen hervor, sondern ebenfalls Mischwesen, ähnlich den Chimären und Monstern, die sie auf diesem Weg besiegen müssen: Es

entstehen Hybride - nicht im Sinn von abgewerteten oder aufgewerteten Bastarden oder Mischlingen, sondern DIE ZUKÜNFTIGE ART. Es ist zu bescheuert konservativ zu denken, dass die alten Formen die maßgeblichen bleiben werden. Umso lieber kann man sie haben, ihre Schwächen einsehend. Denn auch die neuen Formen haben Schwächen und werden bald die alten sein.

(Cotten 2018, 73)

>Prosa`steht für die Unvollkommenheit, das selbstbewusst Unreine, das Halbpatzige, das auf diesen Wegen entsteht, weder ganz alt noch durchwegs neu.

Cotten stellt eine Verbindung zwischen Prosa und spekulativer Philosophie nicht nur dadurch her, dass sie sich fragt, ob die Monster bspw. im Abenteuerroman diejenigen der Welt oder des Bewusstseins sind. Sie tut dies auch, indem sie die Frage nach den Wirkungen des weltbegründenden konstruktiven Anspruchs sowohl der Philosophie wie auch der Prosa stellt. Wenn Herder oder Jean Paul dem Idealismus vorrechnen, dass seine Philosophie auch nicht weiter komme als Romane bei der Frage, ob die Konstruktion der Welt aus dem Ich 
heraus bei der Chimäre stecken bleibt, ${ }^{10}$ dann wird umgekehrt der Anspruch deutlich, dass, wie Ralf Simon (2013, 287) es formuliert hat, »die großen Prosatexte hinsichtlich ihrer weltkonstruierenden Architektonik einen Anspruch stellen, der demjenigen der großen Philosophien ebenbürtig ist «. Aber beide, Philosophie wie Prosa, handeln sich, so Cottens Hinweis auf die »Langstreckenprobleme», dabei auch die gleichen statischen Schwierigkeiten ein. Sie attestiert sowohl der Philosophie wie auch der Literatur, dass in ihnen Chimären als Chimären zur Welt kommen, mit dem Unterschied, dass dies in der Philosophie ein Mangel und in der Literatur eine Stärke - siehe >zukünftige Art` - ist.

Anschaulich wird dies in der Denkoperation eines Paralleluniversums, die der Erzähler Zladko in Cottens Erzählsammlung Lyophilia (2019) durchführt: Er stellt sich - inspiriert von einem dieser gewollt abschreckenden Fotos auf Zigarettenschachteln - ein Universum vor, in dem »alle amputierten Gliedmaßen, alle gescheiterten Projekte« leben. Doch wenn dies auch >nur« in der Vorstellung geschieht - der »Schock« über die Vorstellung ist »echt«, denn »[s]pätestens jetzt, wo ich es mir ausmale, existiert dieses Universum, sofern man den Phantasmata der spekulativen Denkernnnie folgt«. (Cotten 2019, 100) Die daraus resultierende Schwierigkeit besteht darin, dass man »freundlich, verantwortungsvoll« beim Denken sein muss, d.h., »nie etwas denken, was besser nicht wäre, und nie, nie, nie in der Mitte von einer Vorstellung aufhören!« (Cotten 2019, 101) (Denn dann hätte man ja wieder eine chimärische Welt). Doch es ist so schwierig, an diesem Vorsatz festzuhalten, weil die Welt bereits monströse Züge hat, in der mehrere Ablenkungen gleichzeitig gejagt werden müssen: „Es ist ein Kampf mit mehreren Gegnern (Hydra sagt man da).« (Cotten 2019, 101) Die Freiheit, alles denken zu können, wird hier nicht als unendliche Möglichkeit begriffen, sondern stellt ein Problem dar, das mit der Aufgabe der Konzentration und Fokussierung verbunden ist und Verantwortung mit sich bringt. Wie Susanne Lüdemann $(2019,312)$ gezeigt hat, besteht die politische Dimension von Prosa darin, angesichts ihrer Ungebundenheit die Selbstbindung als eine Frage der Verbindlichkeit und Verpflichtung zum Gegenstand der Reflexion zu machen.

10 Simon 2013, 286: „Eines der Hauptbeweisziele des Idealismus besteht in dem Nachweis, dass die Konstruktion der Welt aus dem Ich heraus nicht bei bloßen Chimären stecken bleibt, sondern tatsächlich zur Welt findet. Der Vorwurf, die philosophische Konstruktion sei nur Bild oder nur Literatur, zielt daher auf den wunden Punkt der idealistischen Epistemologie. Seitdem Kant seinen Ausgang bei dem Problem nicht-empirischer, wahrheitsfähiger Referenz, also bei der Frage, wie es denkbar sei, dass ein Gegenstand überhaupt referenzfähig sein könne, gefunden hat, wehren die Theorien den Fiktionalitätsverdacht ab, während die antiidealistische Opposition (Herder, Jean Paul) meint, es dem Idealismus genüsslich vorrechnen zu können, dass er auch nicht weiter gekommen sei, als die Romane es vermögen.« 
Wenn Zladkos Geliebte Ganja auf dessen Schreck über die Realität seines monströsen Paralleluniversums und seinen damit einhergehenden Übereifer mit dem Kommentar antwortet - »Sind wir nicht alle das Produkt nachlässiger Denkernnie« (Cotten 2019, 101) -, dann verweist sie darauf, dass wir gar nicht anders können: Wir sind selbst das von uns geschaffene Paralleluniversum, ausgestaltet von unserer eigenen Nachlässigkeit beim Denken, Gehen, Schreiben. Man ist um ein von Cotten rehabilitiertes Un-Wort der Literaturwissenschaft zu benützen - >beeinflusst ` von den eigenen unausgegorenen Vorstellungen. ${ }^{11}$ Bei allem Verantwortungsbewusstsein ist die Idee eines Perfektionismus gerade diesbezüglich umsonst. Das Problem ist weniger, wie Goethe im Brief an Schiller vom 17. August 1797 meinte, die »millionenfache[] Hydra der Empirie« (Goethe FA II.4, 391), sondern die eigene Tendenz zur Nachlässigkeit und ihre Rückkopplungseffekte. Die politische Verantwortung beim Nachdenken über das Leben wie über Prosa besteht denn auch darin zu fragen: Wenn ihm und ihr alle Möglichkeiten offenstehen - wie weit kommt man, wenn man letztlich immer (wieder) im Sumpf der eigenen Vorstellungen landet? ${ }^{12}$

Literarische Prosa mag ein der Philosophie ebenbürtiger Anspruch einer »weltkonstruierenden Architektonik« sein (Simon 2013, 287), sie ist aber - im Gegensatz zur philosophischen Prosa - eine durchaus selbstbewusste Konfrontation mit der eigenen Nachlässigkeit, die das Chimärische und Monströse, das dem Ornamentalen immer als Verdachtsmoment inhärent ist, berücksichtigt, es ebenso passiv einkalkulierend wie aktiv hervortreibend. Bei Cotten ist, um im architektonischen Bild zu bleiben, jeder Satz ein Palast, aber »wie es bei Palästen ist, verliert man bald den Überblick im Versuch, die Regale zuzuschneiden«, Sätze »bauen sich erst als die unsichtbar gestapelten Wege auf «. ${ }^{13}$ Cottens Architektonik der Prosa ist ein vielfach in sich verschachteltes, labyrinthisches Gebilde, ganz denen ähnlich, wie sie Viktor Schklowski benutzt, um sein Konzept der Ornamentalen Prosa, so der Titel eines seiner Essays, zu charakterisieren: Auf der Basis eines `Sujets`, das auf die einfache Linie, »ein Mensch lebt, wächst und wird alt«, reduziert ist, bewegt »sich der vielstöckige Überbau der metaphorischen Reihen. Diese Reihen, wir stellen sie uns als Häuser vor, sind

11 So heißt es in Lyophilia auch als Konsequenz daraus: „Ich muss aufhören mit diesem Paralleluniversumscheiß, die Vorstellungen beeinflussen mich ja.« (Cotten 2019, 103)

12 Zum Verhältnis Sumpf - Prosa vgl. weiter unten.

13 Cotten 2019, 102. Das ganze Zitat lautet: »Jeder Satz ist ein Palast. Und wie es bei Palästen ist, verliert man bald den Überblick im Versuch, die Regale zuzuschneiden. Kommt um die Ecke und war für Stunden verschwunden. Die Sätze bauen sich erst als die unsichtbar gestapelten Wege auf. Und die bauen sich erst auf, wenn eine Melodie gefunden ist, und die Melodie ist erst gefunden, wenn -«. 
untereinander durch schmale Hängebrücken verbunden. Durch ihr Voranschreiten schafft die Fabel den Vorwand für neue Reihen, die bei ihrem Entstehen sogleich mit den bereits bestehenden verbunden werden.« (Schklowski 1987, 94f.) Das Bild der Perlenschnur, auch wenn sie beweglich ist, reicht nicht aus, um Prosa zu charakterisieren. Die Wege und Vorstellungen, die man (im Leben wie im Text) geht, durchkreuzen die Linearität des zeitlichen Ablaufs und es entsteht ein Muster, in dem man schnell einmal die Orientierung verliert. ${ }^{14}$

\section{Lineaturen der Literatur}

Dass Zladkos Lieblingsbeschäftigung darin besteht, mit seinem Synthesizer Module zu kreieren, die er dann in die »Loop-Machine $"$ nimmt, ${ }^{15}$ und sich damit tatsächlich zusammen mit Ganja in das zuvor ınur` vorgestellte Paralleluniversum katapultiert - hier sind die Gefahren, etwa eines imaginierten Karamelltsunamis, tatsächlich real -, ist ein Indikator dafür, die Wirkungen der Rekursivität auch im Bereich des Visuellen und mit Blick auf den immer wieder konstatierten Zusammenhang von Prosa und Schrift/Schriftlichkeit zu untersuchen (Barck 2003, 88).

Schklowski entwickelt das Bild der Hängebrücken, unmittelbar nachdem er auf die besonderen grafischen Strukturen in Andrej Belyis Aufzeichnungen eines Sonderlings hingewiesen hat (Abb. 1).

Die Auflösung der vermeintlichen Gradlinigkeit von Prosa provoziert zunächst, was Jean Paul als Wendung des »Blicks von der Sache [...] gegen ihr Zeichen hin« (vgl. hierzu Simon 2013, 256f.) beschrieben hat - es ist ein Blick, der sich auf die Materialität des Zeichens erstreckt und dort, um mit Aleida Assmann zu sprechen, einen Prozess komplexer, wilder Semiose in Gang setzt (Assmann 2015; Simon 2013, 208). Die Plurifokalität wird als Charakteristikum von Prosa sichtbar (Simon 2018, 417), indem die ornamentalen Strukturen digressiver (d.h. von stringenter chronologischer Handlung abweichend) Prosatexte darauf hin gelesen werden, wie sie ihre eigene Struktur theoretisieren bzw. zur Darstellung bringen. Michel Foucault bemerkt anhand von Paul Klees Bildern, dass sie eine Auflösung eindeutiger Ordnung bzw. Richtung »von der Form zum

14 Inka Mülder-Bach (2019b, 226) hat formuliert, dass Prosa »seit der Antike auf eine Freiheit hin akzentuiert [wird], die einerseits Bewegungsspielräume eröffnet, andererseits nach Formungen und Selbstbindungen verlangt, um nicht zur Orientierungslosigkeit zu führen«. Mit Cotten kommt allerdings in den Blick, dass sie gerade in den internalisierten (Selbst-)Bindungsmechanismen wiederum die Orientierung verliert.

15 Cotten 2019, 188. Zum Loop: Baumgärtel 2016. 


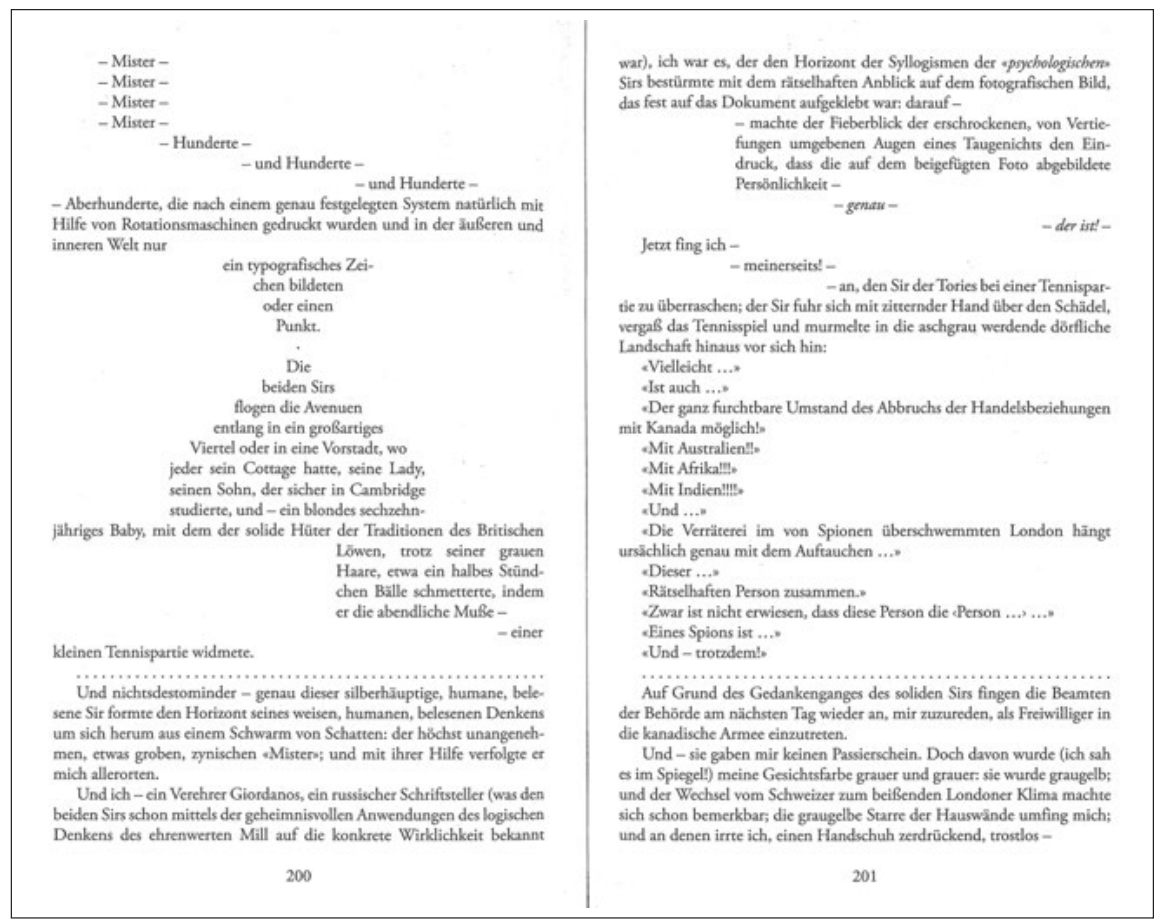

Abb. 1: Belyi, Andrej: Aufzeichnungen eines Sonderlings. Aus dem Russ. übertr. und hrsg. v. Christoph Hellmundt. Dornach 2012, $200 \mathrm{f}$.

Diskurs oder vom Diskurs zur Form« implizieren: Sie bilden einen »ungewissen, umkehrbaren, schwebenden Raum (zugleich Blatt und Leinwand, Fläche und Masse, kariertes Heft und parzellierte Erde, Geschichte und Karte)«, in dem die "Komposition der Figuren und die Syntax der Zeichen« gleichzeitig möglich sind. ${ }^{16}$ Visuell evident wird dies bspw. in der 1938 entstandenen Zeichnung Vortrag über die Spirale (Abb. 2), wo der Körper des Vortragenden zugleich die Spirale ist und hervorbringt.

Was Foucault für diese Bilder bemerkt, gilt auch für `Muster Prosa`, die mitunter durch grafische Elemente die Aufmerksamkeit auf ihre Schrift-Bildlichkeit lenkt, um überhaupt die sprachliche Bewegung sichtbar werden zu lassen. ${ }^{17}$

16 Foucault 1997, 26f. Zu den gegenläufigen Richtungen von Prosa und ihrem Verhältnis zur Schriftlichkeit Simon 2018, 426 f.

17 Vgl. auch den knappen Hinweis von Ralf Simon (2018, 417): »Typographische Experimente lösen die Kontinuitätsidee des Textverlaufs auf und führen auf die Reflexion des immanenten Textualitätsprinzips.« 


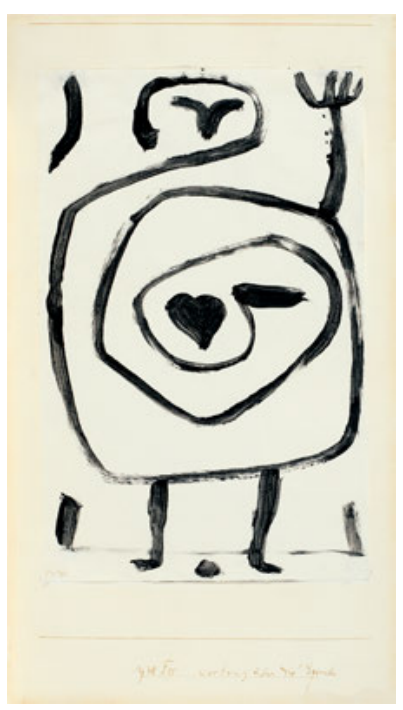

Abb. 2: Klee, Paul: Vortrag über die Spirale, 1938, 75, Kleisterfarbe auf Papier auf Karton, 27,9 × 17,8 cm, Standort unbekannt @ Zentrum Paul Klee, Bern, Archiv.

An der Schnittstelle von Linie, Ornament und Schrift wurde in den letzten Jahren viel gearbeitet, ${ }^{18}$ und ich beschränke mich auf einige Hinweise: Entscheidend für eine Theorie von Prosa, die sich auf die Lineaturen der Literatur, also die Linien, anhand derer Prosa sich selbst reflektiert, bezieht, ist die Emanzipation der Linie vom Kontur und damit von der Bezeichnung, wie sie sich im achtzehnten Jahrhundert vollzieht. Für Paul Klee ist die saktiveく Linie zu einem Akteur geworden, sie bildet nicht ab, sondern sie macht "sichtbar «: »Eine aktive Linie, die sich frei ergeht, ein Spaziergang um seiner selbst willen, ohne Ziel: das agens ist ein Punkt, der sich verschiebt. ${ }^{19}$ (Abb. 3)

Klee theoretisiert in seiner Formenlehre, wie ein in Bewegung versetzter Punkt eine Linie zeichnet, die sich zu Figuren und Mustern ausbilden kann. Seine Darstellungen vollziehen Bewegung und verschieben dadurch den Fokus von der statischen Form des Bildes zur Prozessualität - dem Künstler liege doch, so merkt er im Jenaer Vortrag von 1924 an, »mehr an den formenden Kräften als an den Form-Enden« (Klee zit. n. Kain 1999, 35). Die Grundlagen, auf denen dieses Wortspiel basiert, bilden William Hogarths Analysis of Beauty (1753) und Laurence Sternes Tristram Shandy (1759-1767) (Bedenk 2004, 48). Hogarth ist für >Muster Prosar deswegen von zentraler Bedeutung, weil er, wie Gerhard von Grae-

18 Mersmann 2015; Driesen 2012; Krämer und Cancik-Kirschbaum 2012; Boehm 2007; Krämer und Bredekamp 2003.

19 Klee 1925, 6. Vgl. hierzu Bonnefoit 2009. 
Eine aktive Linie, die sich frei ergeht, ein Spaziergang um seiner selbst willen, ohne Ziel. Das agens ist ein Punkt, der sich verschiebt (Fig. 1):

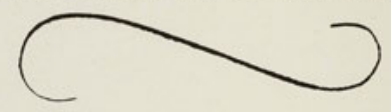

Fig. 1

Dieselbe Linie mit Begleitungsformen (Fig. 2 und 3):

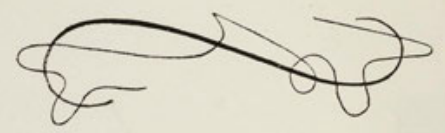

Fig. 2

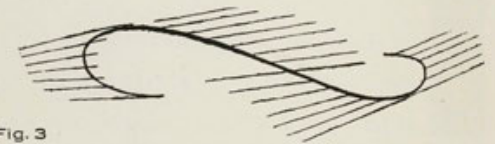

6

Abb. 3: Klee, Paul: Pädagogisches Skizzenbuch. München 1925, 6.

venitz gezeigt hat, räumliche Bewegungen in ornamentale Grundrisslinien auf der Fläche übersetzt, d.h., die Dreidimensionalität auf zwei Dimensionen plus die Zeit des Nachvollzugs in der Betrachtung reduziert (Graevenitz 1994, 42; Bedenk 2004, 48, 72). Die freischwingenden Bewegungen der Schlangenlinie, wie 
sie auch als Spuren des Menuett-Tanzes auf dem Boden sichtbar werden (Abb. 4), verkörpern nicht nur das Prinzip der Schönheit, sondern »das Besondere« dieser Linien besteht in der »Verwicklung der Form « und der Verlockung des »Auge[s] zu einer spielerischen Weise des Verfolgens« (Hogarth 1995, 6; Bonnefoit 2004).

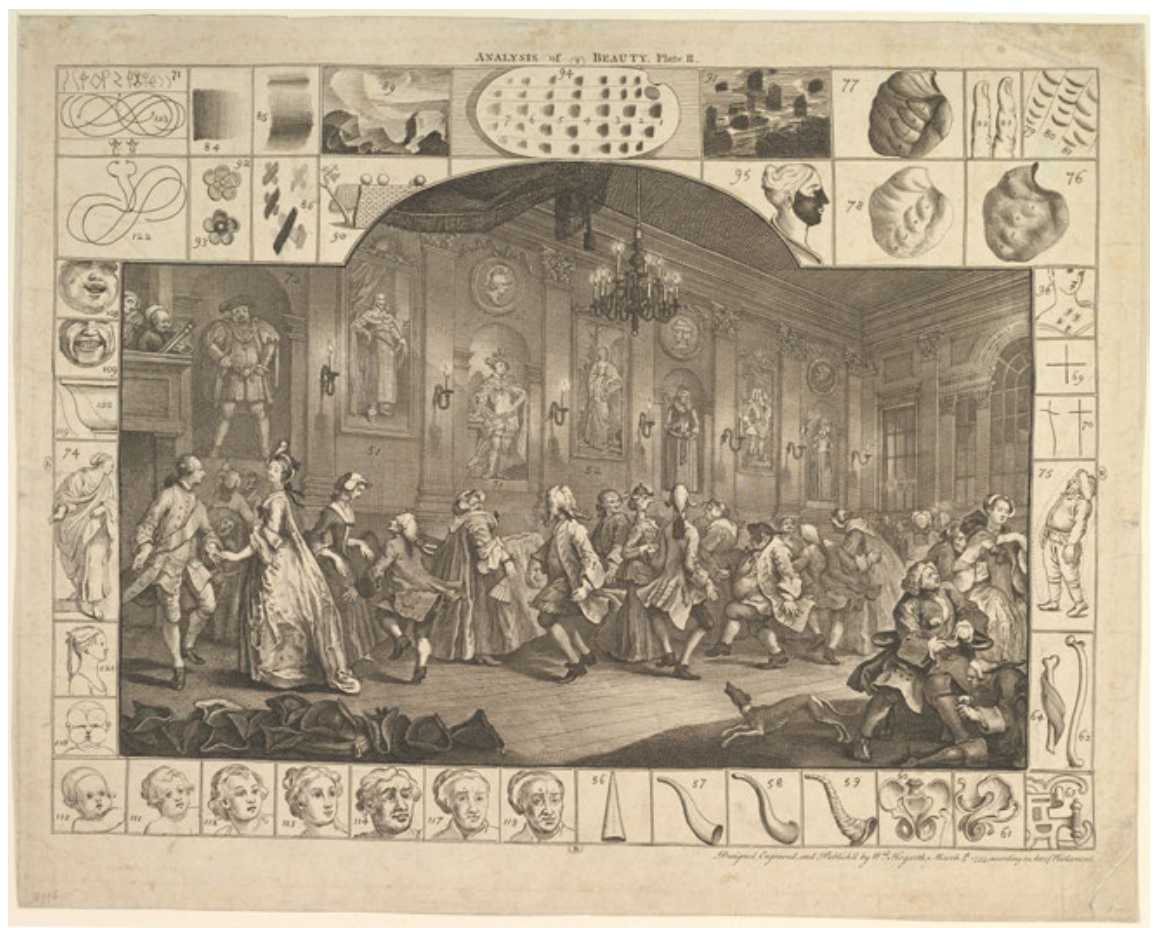

Abb. 4: Hogarth, William: Analysis of Beauty. London 1753, Taf. 2, $42.5 \times 53.5 \mathrm{~cm}$. (c) TheMet, Gift of Sarah Lazarus, 1891.

Hogarth ermöglicht die Entkopplung der Linie von der Nachahmung, wie sie die Anthropologie und Ästhetik des Klassizismus bis zur Moderne bestimmen wird - von John Flaxman bis Wassily Kandinsky. ${ }^{20}$

Auch Laurence Sternes Roman Tristram Shandy, der unmittelbar auf Hogarth reagiert, steht für eine solche Emanzipation der Linie - die Schnörkel seiner stän-

20 Rosenblum 1967. Zu diesen Tendenzen der Kunst(theorie) um 1800, die auf die moderne Abstraktion vorausweisen, gehört natürlich auch die romantische Diskussion rund um die Arabeske, Hieroglyphe etc. 
digen Digressionen werden in den berühmten Plotlines sichtbar, das Hogarth'sche Schönheitsideal damit freilich karikierend (Abb. 5) (Bedenk 2004, 47 f.).

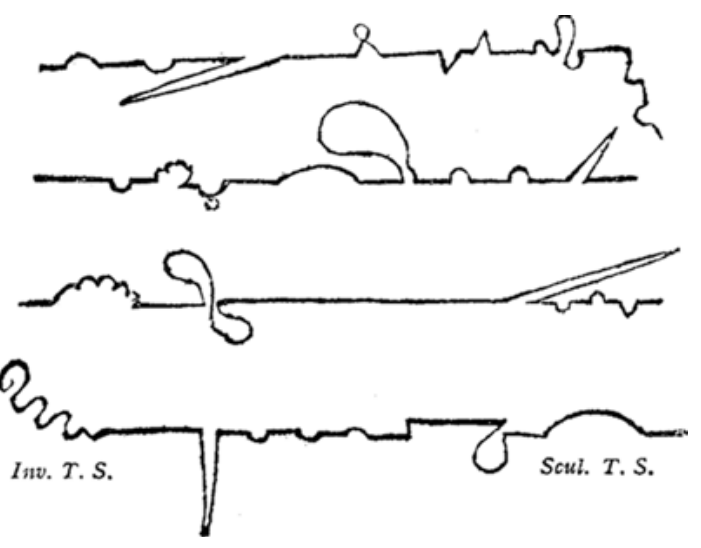

Abb. 5: Sterne, Laurence: The Life and Opinions of Tristram Shandy, Gentleman. London 1759-1767, Bd. 6, 152.

Der Effekt von Prosa ist auch hier, dass die einfache Form >verwickelt ` wird und man spielerisch die Verwicklungen bei der Lektüre nachvollzieht. Dabei geht es auch hier darum, dass aus den Abweichungen Muster hervorgehen, Digression also strukturbildend ist. Der Tristram folgt, so Jochen Bedenk, einem »fragmentarisch-kaleidoskopartige[n] Erzählen, das seine innere Kohärenz wie bei Hogarth aus der Herausarbeitung von wiederkehrenden und für den Leser wiedererinnerbaren Motiven erhält« (Bedenk 2004, 48). Einerseits rollen Leben und Text im Tristram unerbitterlich $a b$ ovo voran; gleichzeitig gerät der Text mit seinem eigenen Voranschreiten in Konflikt, wenn er all die gleichzeitigen Ereignisse und ihre unterschiedlichen Temporalitäten integrieren und aufeinander abstimmen möchte. Wie in keinem zweiten Text steht Prosa hier für die Ironie, dass, wer sich dem Ideal einer teleologischen Ausrichtung verschreibt, sich unweigerlich in sich selbst verheddert.

Dass Ann Cotten gleich zu Beginn von Lyophilia auf diesen Roman als einen zentralen Intertext verweist, ist kein Zufall. Es wird die Perspektive von ausserirdischen Kolonisatoren auf die Erde eingenommen und bezüglich dieser Anfänge gefragt: »Wie soll man das erzählen?« (Cotten 2019, 7) In den Anekdoten und Sagen, die bezüglich dieses frühen Stadiums der menschlichen Entwicklung kursieren, kommen drei mögliche Auslegungen über die menschliche Art vor: Die erste sieht in den Menschen »langfristige[] Denkernnnie«, die dritte verbindet sie mit dem Wort »Easy« und dem Drang nach Spaß und die zweite sieht als zentrale 
Motivation zur Entwicklung den »Wille[n]«. Hier kommt auch der Vergleich der Erde mit dem »Garten des Onkels von Tristram Shandy«, hat sich doch diese letztgenannte Gruppe der Menschen »seither in Milliarden von Scharmützeln und sonstigen Tätigkeiten [...] ausgetobt« (Cotten 2019, 8). Der Verweis auf Sterne beschränkt sich jedoch nicht auf die Kriegsobsession von Toby, sondern bezieht sich vor allem auf die Wiederholung von Modellen in immer kleiner werdendem Format, wie sie unmittelbar darauf als explizite Reflexion auf die Gattung der Anekdote folgt:

\begin{abstract}
Dabei ist schon die Form der Anekdote oder Sage eine Art Selbstmimikry. Kringel, eine häufige Figur der notwendig im Sand verlaufenden, also schiefgehenden (fraktalen: also wachsend wie abbrechend so oder so sich verlierenden) Fortentwicklung dieses durch die Evolution als auf einem Fleck bleibend auf ständige Kollision mit sich selbst getunten Systems.

(Cotten 2019, 8)
\end{abstract}

Der Text über die vermeintlichen Uranfänge stellt hier gleich zu Beginn fest, dass Erzählungen über Anfänge in Form von Anekdoten oder Sagen bereits systemstabilisierend sind. ${ }^{21}$ Denn man kann ja gar nicht anders, als sich selbst nachzuahmen. Der Prozess der Selbstmimikry in Prosa entspricht einem fraktalen Muster, das sich selbst ähnlich wird, während und indem es immer fortläuft. `Muster Prosa` macht also sichtbar: Bereits während man zu schöpfen meint, betreibt man Legendenbildung.

Nach dem gleichen Prinzip funktioniert auch die Prosa von Robert Walser ein weiterer Schriftsteller, der die Affinität von Gehen und Schreiben auf die Spitze getrieben hat, der - von Susan Sontag 1982 im Vorwort einer Anthologie als »Paul Klee in prose« bezeichnet (Walser 1982, VI) - die Lineaturen der Literatur nicht von den »Form-Enden« sondern von den formenden Kräften her in den Blick nimmt. Walter Benjamin hat bei ihm eine »zumindest scheinbar, völlig absichtslose[] und dennoch anziehende[] und bannende[] Sprachverwilderung" konstatiert, die »alle Formen« hat, »mit Ausnahme einer einzigen. Nämlich dieser einen geläufigsten, der es auf den Inhalt ankommt, und sonst auf nichts." (Benjamin 1991, 325) Walsers sarabeske Prosa lässt sich nicht auf eine bestimmte Form ein, die wilde Semiose sprengt als Verwilderung die herkömmliche Verbindung von Form und Inhalt. ${ }^{22}$ Doch auch bei ihm bilden sich in den ornamentalen Digressionen Rückkopplungseffekte heraus, und die Verstrickung in die eigenen Muster kommt in den Blick. So bemerkt auch Benjamin, dass sich Walser

21 Vgl. zur Anekdote als prekäres Philosophieren aus dem Rückblick den Beitrag von Rüdiger Campe in diesem Band.

22 Vgl. zur Verbindung zwischen Klee und Walser bspw. Scheffler 2010, $312 \mathrm{ff}$. 
»bacchisch mit Sprachgirlanden [kränzt], die ihn zu Fall bringen. Die Girlande ist in der Tat das Bild seiner Sätze. Der Gedanke aber, der in ihnen daherstolpert, ist ein Tagedieb, Strolch und Genie wie die Helden in Walsers Prosa." (Benjamin 1991, 326) Walser schmückt sich mit Girlanden, über die er sodann beim Rumstreunen durch die Gegenden und im Text stolpert: Exzentrik und Verstrickung fallen wiederum zusammen. Die Forschung hat herausgearbeitet, wie die ornamentalen, bisweilen >labyrinthischen « Muster auf der syntaktischen Ebene beschrieben werden können, etwa indem der Reim bisweilen als Antriebsmoment von Zeile zu Zeile funktioniert, bis ein neuer Gedanke und ein neuer Klang einen weiteren Zweig entstehen lässt. ${ }^{23}$ Gezeigt wurde aber auch, wie diese Muster die Gesamtstruktur dieser Texte betreffen, die von Parallelismen, Reprisen und Wiederholungen gekennzeichnet sind, ${ }^{24}$ und wie sie den Schreibprozess selbst bestimmen - Walsers berühmtes »bleistifteln, zeichnelen und gfätterlen" (Walser zit. n. Andrea 1997, 80) -, weil es »so hübsch« ist, »die Zeilen mit zierlichen Buchstaben auszufüllen « (Walser zit. n. Andrea 1997, 11), bis, so die Formulierung von Susanne Andres (1997, 160), eine »lückenlos ornamentierte Fläche« entsteht. Die Ausbildung von Mustern macht die Selbstbindungstechniken von Prosa reflexiv zum Gegenstand, und auch hier handelt es sich nicht um einen reinen Ästhetizismus, sondern es geht um die damit verbundenen politischen Implikationen. Nicht umsonst greift Cotten ganz zu Beginn ihrer Poetikvorlesung Was geht auf Walsers Der Spaziergang zurück, einen Text, den sie als den "naheliegendsten « in ihrem Kontext, aber doch wegen seiner »kometengleichen Hyperbole als nicht ganz einfach zu zitierenden Text« bezeichnet: ${ }^{25}$ Die Erklärungen des Erzählers über die Zweckdienlichkeit, ja Notwendigkeit des Spazierengehens für ihn als Schriftsteller seien, so Cotten, mit dermaßen vielen Superlativen und anderen »Blüten des Übertreibungsstils" sowie mit der "Klinge der Überaffirmation" gespickt (Cotten 2018, 17), dass sie die Wahrheit auf fast indiskrete Weise aussprechen, sie aber dabei gleichzeitig wieder in der Ironie verstecken und dadurch erst subversiv wirken, sich dem Paradigma der Notwendigkeit grundsätzlich entziehend.

Der Konnex von Gehen, Schreiben und Musterbildung im Zeichen von ıProsa ließe sich an vielen weiteren Beispielen aufzeigen, besonders produktiv ist aber Peter Handke, auf den Cotten in der eingangs zitierten »Reiterei durch das Bewusstsein« Bezug genommen hat. Seine Arbeit an einem neuen epischen Schrei-

23 Andres 1997, 84-86, zum >Labyrinthischen<, 156.

24 Albin Zollinger beschreibt die Komposition in Der Gehülfe als »wohlbewußte Technik des Parallelismus, der Reprise, des Umgangs im Kreise oder, richtiger gesagt, in der Spirale« (Zollinger 1978, 137).

25 Cotten 2018, 13. Vgl. ausführlicher zur Figur der Hyperbel bei Cotten: Keller 2020b. 
ben, das tatsächlich in enger Verbindung mit der mittelalterlichen Epik des Artusromans steht, führt zu grundlegenden Überlegungen zu Prosa als `Meta-Literatur`, die ihre verschiedenen Bindungsformen als Lineaturen der Literatur reflektiert: Gegenüber der `Linie` des Bildungsromans integrieren Handkes jüngere lange Prosatexte verschiedene Gattungen, die sie in ihren Mustern ausstellen. ${ }^{26}$ Wie ich an anderer Stelle gezeigt habe, stellt Handke dem Zickzack des Dramas die geschwungene Schönheitslinie des Märchens und dem Mahlstrom des Romans à la Proust die ausgreifende Spirale des epischen Erzählens entgegen (Keller 2020a). Auch bei ihm stehen bei aller Digression stets die Wiederholung und damit die strukturbildende Funktion von sich zu Mustern ausbildenden Linien im Zentrum. Die Bedeutung von Rekursivität zeigt sich bereits an den emphatisch wiederkehrenden, in sich selbst gefalteten Sprachgebärden »hin und her«, "auf und ab«, »kreuz und quer«, die allein schon eine Steilvorlage für Giorgio Agambens Hinweis auf die bustrophedische Bewegung von Prosa bilden. ${ }^{27}$ Episches Schreiben (und man könnte hier als Synonym wiederum Prosa einsetzen) ist laut dem Journal Am Felsfenster morgens deswegen so herausfordernd, »weil es stetig zugleich Rückweg, Rückweg ganz zurück ist und Weiterweg, Weitergehen, ständiges erfordert« (Handke 1998, 179). Auch bei Handke stiftet Prosa eine Unordnung zwischen den eindeutigen Richtungen von Form und Diskurs sowie von Erzählen und Reflexion.

Wenn für eine Theorie der Prosa beide Richtungen beachtet werden sollen, so soll bei der fortlaufenden Dekonstruktion, die die Texte all dieser erwähnten Autor`innen laufend selbst betreiben, mit der Frage, was dabei passiert, die politische Dimension einer solche Theorie auch noch einmal mit Blick auf Cotten diskutiert werden. Ich setze erneut bei der Frage an, auf welche chimärische, >zukünftige Art` die Musterbildungen ihrer Lineaturen der Literatur zielen. Lyophilia ist nicht umsonst ein Hybridtext, kein Roman, aber mehr als eine Sammlung von

26 Zum Muster bei Handke vgl. Honold 2017, 89: »Ein solches `Muster der durch das Reisen erzeugten Raumwahrnehmung stiftet nun allerdings eine andere, komplexere Art von Ordnung, als es die >Linie des tradierten Bildungsromans und seines sprichwörtlichen $>$ Fadens der Erzählung` getan hatte, wie Robert Musil diese Darstellungskonvention einmal spöttisch titulierte. Dem Muster ist die Knotenstruktur von Verzweigungen eingeschrieben, es ist auf die simultane Kopräsenz von alternativen Möglichkeiten ausgelegt, die als Bifurkationen im Raum oder als Rochaden in der Zeit erzählt werden können. Im Muster sind die vergangenen und die künftigen Wegstrecken, die indikativischen und die konjunktivischen Aufenthalte in einem Bild vereinigt. Man muss also nicht unbedingt die hinzugefügte Kartenskizze des Klappentextes bemühen, um im Kurzen Brief einen dem Erzählvorgang inhärenten figurativen Gestaltungswillen zu erkennen."

27 Zum >Hin und Her vgl. Keller 2020a, sowie als Figuration von Prosa, jedoch mit Blick auf Goethe: Mülder Bach 2019b. Zur ১bustrophedischen` Prosa vgl. Agamben 2003 sowie, darauf Bezug nehmend, Simon 2018, 426. 
Erzählungen; er realisiert die von Hélène Cixous für das eigene Schreiben emphatisch formulierte dekonstruktive Einsicht »il n'y a pas de genre« (Cixous 2018, 68) - die auch als eine Einsicht in >Prosar gelten kann - bis in die grafische Gestaltung hinein. Gleich auf der ersten Seite stören japanische Schriftzeichen den Lesefluss der durchschnittlichen deutschsprachigen Leser`innen, lenken den Fokus auf das Visuelle und die grundsätzliche MehrSprachigkeit von Prosa: ${ }^{28}$

Die Außerirdischen also, die uns gegenüber die Erscheinungsform 宇宙人 gewählt haben, erzählen sich verschiedene Anekdoten über die Frühzeit der Kolonisation. Eine davon geht so:

Wie soll man das erzählen?'

Damit führt der Text sogleich die Grenzen des Les- und Verstehbaren vor Augen, er wirft hermeneutische Fragen auf, aber er geht auch ganz selbstverständlich von der Notwendigkeit des Codeswitching - sowohl zwischen Zeichen als auch zwischen Sprachen - in einer futuristisch anmutenden Gegenwart aus. Dies betrifft >natürliche` Sprachen ebenso wie ihre technisch-mediale Veränderungen und auch die Fehleranfälligkeit beim Codeswitching. So gibt das zweite Kapitel, das sich um »Weltspionageakten« dreht, das Resultat einer irritierenden Internetrecherche (»Wikileaks«) wieder (Cotten 2019, 9):

Äh August, äh, 12ter, äh 2011 Äh äh John äh Gibbonsäh Stratfor äh

221 äh Westäh 6 ste äh Straße äh Steäh 40oäh AustinähTexas, Äh 78701 äh

PER ÄH E-MAIL: Äh äh Gibbons@stratfor.com äh Äh Lieber äh John, äh

Bitte äh lass äh Don äh die äh Anmeldung äh unterschreiben äh wo äh markiert äh ist. Äh äh äh

Sobald äh die äh Anmeldung äh unterschrieben äh istäh, bitteäh schickeäh sieäh miräh per äh Email äh oderäh Fax. Äh zusammen äh mit äh den äh folgenden äh unterstützenden äh Dokumenten: ähäh

28 Cotten 2019, 7. Die Zitate und Zeichnungen aus Lyophilia (Cotten 2019) werden hier und im Folgenden als Abbildungen der Druckseite behandelt. Ich danke Ann Cotten, dass sie mir die digitale Version des Textes zur Verfügung gestellt hat. Vgl. zum Zusammenhang der Mehrsprachigkeit den Beitrag von Till Dembeck in diesem Band. Hier ist Mehr-Sprachigkeit auf die Bildlichkeit auch als `mehr als Sprache` zu verstehen. 
Der Kommentar dazu lautet: »Es klingt wie ein sprechender US-Amerikaner oder Engländer, und ich frage mich, ob es wohl von der Verschlüsselung oder vom Diktat kommt.« (Cotten 2019, 9) Hat hier das Programm versagt, das automatisch gesprochene Sprache in Schrift transformieren soll? Oder werden hier die Fehler sichtbar, die beim Versuch passieren, Sprache zu ver- und wieder $\mathrm{zu}$ entschlüsseln? Für Lyophilia ist eine solche medientheoretische Weiterentwicklung einer Derrida'schen écriture deswegen zentral, weil so das auf der inhaltlichen Ebene verhandelte Thema - nämlich wie Systeme und ihre Muster präsent sind, sich aber unserer Wahrnehmung entziehen - über eine sprachliche Irritation in den Bereich der Wahrnehmung geholt wird. Zugleich geht es darum, auch die Strukturbildung durch und in der Sprache zunächst zu reflektieren, bevor der Text ins Erzählen kommt. Lyophilia verschleiert den Zugang zu sich selbst, schiebt das Erzählen, das Schreiben, das Lesen und damit auch die Sinnkonstruktionen im Erzählen hinaus und richtet den Fokus zunächst auf die Materialität der Sprache und ihre Muster.

Aber auch später in den erzählerischen Passagen lenkt der Text immer wieder den Blick von der Bedeutung auf das Zeichen in seiner Materialität und stellt so die Paradoxa der eigenen Medialität mit Komik, die bisweilen zu Slapstick gesteigert wird, aus (Cotten 2019, 320):

"Horatio «, flötet Elektras Stimme wieder in die Garage.

Die gewellte (Schönheits-)Linie scheint den dramatischen Effekt dieser Dialogpassage $\mathrm{zu}$ verstärken, wird doch die Intonation, die der schriftliche Text sonst nicht wiederzugeben vermag, integriert. Doch geschieht dies durch die ıÜbersetzung، in ein grafisches Element, und dieser unvermittelte Einbruch des Visuellen in die sprachliche Struktur bewirkt einen Bruch mit der mimetischen Illusion, die sich in der Lektüre normalerweise einstellt - man merkt wieder, dass man eigentlich gerade am Lesen ist. Vergleichbar ist die Wiedergabe eines Namens in Spiegelschrift (Cotten 2019, 363):

Wir saßen schon eine Weile da und schwiegen uns an. Sie blickte zur Tür, las meinen spiegelverkehrten Namen am Milchglas - IMAHJZ Id - und drehte den Kopf urlangsam wieder zu mir, als wäre sie vom Teufel besessen und im Begriff, vor 360 Grad nicht haltzumachen. Dann spitzte sie ihre 
Einerseits wird Unmittelbarkeit suggeriert - ich lese genau das, was innerhalb der Diegese die Erzählinstanz liest. Und doch stellt die Spiegelschrift, die besondere Anforderungen an ihre Erkennbarkeit - zunächst denkt man vielleicht, es handle sich um kyrillische Schrift - wie auch an ihre Entzifferung stellt, ein Distanzierungsmoment dar, eine Schwelle, die der Illusion der Lektüre entgegenläuft. Indem zudem die Blickrichtung beim Lesen innerhalb einer Zeile wechselt, realisieren sich der bustrophedische Charakter von Prosa wie auch ihre Mehrstrahligkeit und Plurifokalität (Simon 2013, 257).

So gibt es in dem Text auch Passagen, in denen die bei Prosa normalerweise gefüllten Zeilen aufgebrochen und wie in Belyis Aufzeichnungen eines Sonderlings grafisch angeordnet werden (Cotten 2019, 190):

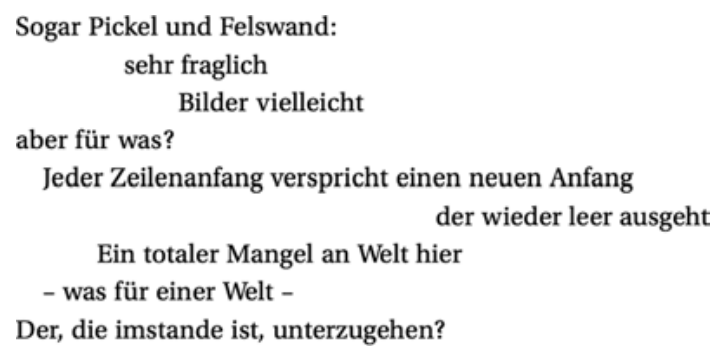

Hier wird der totale whiteout nach dem Eintritt ins Paralleluniversum, als sich nur allmählich einige fragmentarische Bilder und Sätze aus dem Nichts herausschälen, performativ umgesetzt. Dabei kommt auch hier ein Paradox zur Anschauung, macht doch erst die Zeile, die auf den vielversprechenden Anfang folgt, durch ihre Positionierung sichtbar, dass dort tatsächlich eine Leerstelle ist, wobei sie gleichzeitig die Aussage gerade durch diese Positionierung am Ende widerruft. Prosa löst die geschlossene Form und ihre Ordnungen hier Zeile für Zeile und in jeder Zeile auf, gleichzeitig macht sie das Paradox sichtbar, dass gerade auch dadurch sich wieder Musterbildung vollzieht.

Dass es sich dabei keineswegs um Spielereien handelt, sondern (politische) Fragen der sogenannten Weltanschauung auf dem Spiel stehen, zeigt sich auch an einer weiteren grafischen Irritation, die sich in Worten wie »Denkernnnie« zeigt (Cotten 2019, 7). Die Auflösung der Leserichtung, das bustrophedische Prinzip von Prosa, hat sich hier bis in die Anordnung der einzelnen Buchstaben hinein vollzogen: Es handelt sich dabei um das von Cotten inzwischen konsequent angewendete spolnische، Gendering, bei dem, wie es im Impressum von Lyophilia heißt, "[a]lle für alle Geschlechter nötigen Buchstaben [...] in gefälliger Reihenfolge ans Wortende« kommen (ebd., vgl. den Vermerk im Impressum). Auch hier gerät durch die visuelle Irritation zunächst die textuelle Medialität überhaupt in den Bereich der 
Aufmerksamkeit, aber gleichzeitig werden durch die Irritation der Aus-Richtung überhaupt die Welt- und Denkordnungen, die sich gern im Bereich des Unsichtbaren verstecken, sichtbar gemacht - und transformiert: Denn es wird in dieser Neuanordnung der Buchstaben nicht nur Cixous' Aussage, dass es keine Gattungen gäbe, realisiert; es findet ein ganz konkretes, sich auf der basalen Ebene der Buchstaben vollziehendes »rewriting der kulturellen Ordnungsmuster« statt (Simon 2018, 421). Diese Form des Gendering, das sich wohl den Vorwurf einer monströsen >Verunreinigung ‘ der Sprache gefallen lassen muss, bildet affirmativ die chimärischen Wesen der `zukünftigen Art ` - ohne moralischen Zeigefinger, sondern spielerisch mit der Affinität von Prosa zu einer bisweilen grotesken Komik.

Die Hybridisierung und Auflösung von Gattungen realisieren sich in Lyophilia auf allen Ebenen, die Erzählperspektiven wechseln ebenso wie die Figuren durch die einzelnen Texte mäandrieren und instabile Identitäten aufweisen. »Eien« (also: ১Ein` plus ১Eine`) heißt eine Figur, die manchmal auch die ${ }^{\star}$ der Erzähler*in ist, deren Freund mit dem Namen Emile Singular und Plural zugleich ist/sind: »Er legen ein paar Arme um mich, kosten das kurz, dann lassen er sich neben mich fallen und stapeln die Köpfe auf meine Schulter.« (Cotten 2019, 278) Auch bei der Darstellung von Emile besteht das `Zugleich` von Prosa darin, dass es sich um Ernst und Komik gleichzeitig handelt (Simon 2013, 287). Besonders deutlich wird dies bei den beiden Zeichnungen, auf denen er/sie rabgebildet wird/werden (Cotten 2019, 276f.):

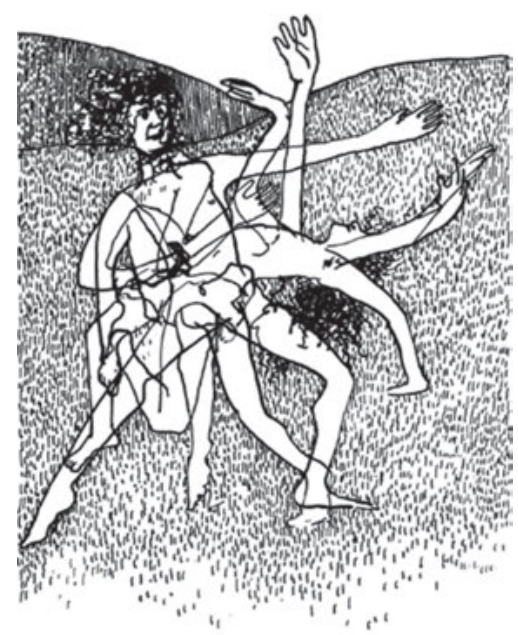

276

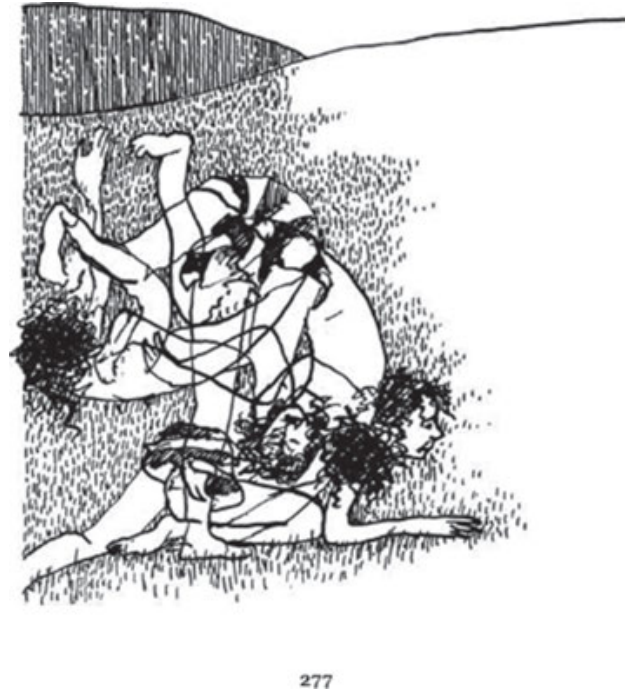


Unabhängig vom Text betrachtet erinnern die Zeichnungen an Bewegungsdarstellungen von Eadweard Muybridge sowie an kubistische Verfahren, mehrere zeitliche Momente in einer Darstellung zusammenzuführen und so Temporalität in das Bildparadigma zu integrieren. Das Slapstick-Moment entsteht dadurch, dass Emile hier - wie Walser, der sich in seinen Sprachgirlanden verheddert - in der eigenen Bewegungsornamentik zu Fall gebracht wird/werden. Wobei die Pointe und damit auch der gleichzeitige Ernst - darin besteht, dass, was wie die Darstellung einer zeitlichen Abfolge aussieht, eigentlich vielleicht doch eine Darstellung der mehreren Gliedmaßen - Stichwort: Hydra - von Emile und also ein >Abbild seiner/ihrer Pluralität ist. Metaphorisch auf das Leben bezogen könnte dies verstanden werden als: Man denkt, dass man sich mit der Zeit verwandelt, dabei ist man immer schon mehrere gleichzeitig - und kommt sich selbst in die Quere. Metaphorisch auf Prosa bezogen könnte formuliert werden: Komplexe Prosatexturen erzeugen Pluralität und Diversität, sie lösen die Richtungen und Ordnungen auf und schaffen eine Dichte, die diesem Knäuel von Linien und Verrenkungen gleichkommt. Prosa realisiert so ihre >zukünftige Artヶ.

Denn vielleicht ist in Cottens Lyophilia eine Prosaform realisiert, wie sie in einer vom Bild dominierten Gegenwart des einundzwanzigsten Jahrhunderts von zunehmender Bedeutung sein könnte. Die Bildwissenschaften haben ihre Aufmerksamkeit schon länger vom Bildobjekt auf den Bildträger verschoben und analog dazu schaffen solche Texte etwas Vergleichbares für die Literatur - bereits die Tatsache, dass im vorliegenden Beitrag die angeführten Textbeispiele als Bild ızitiert` werden, verweist darauf. Gerade indem solche Texte sich viel stärker zum Bereich des Visuellen hin öffnen, als es in herkömmlichen Text-Bild-Relationen der Fall war, findet auch eine Reflexion auf die medialen Eigenheiten der Schrift statt, die zu einer verstärkten Aufmerksamkeit auf die Materialität der Buchstaben führt. Auffallend viele Beispiele der Gegenwartsliteratur in Prosa - Comics und Graphic Novels sind hier nur die medialen Außenränder dieses Phänomens - nutzen die grafische Gestaltung genau in diesem Sinn: Sie treiben das Zugleich von Diskurs und Anschauung auf die Spitze und loten die Möglichkeiten der Lineaturen der Literatur aus, indem sie Form in Bewegung auflösen, um daraus Muster $\mathrm{zu}$ bilden, und indem sie ein Spiel mit Abstraktion, Schriftbildlichkeit, Blick- und Leserichtungen betreiben. ${ }^{29}$ Nicht umsonst nähert sich Jason Lutes' Berlin-Trilogie (Lutes 2018), um nur eine jüngere Graphic Novel in ihrer avancierteren Form zu nennen, an die großen Romane der klassischen Moderne an, mit einem hohen Grad an Autoreflexivität, Serialität des Erzählens, einem unabschließbaren enzy-

$29 \mathrm{Zu}$ diesem Phänomen der Gegenwartsliteratur mit zahlreichen Beispielen: Graf 2019. 
klopädischen Umfang und einer Polyphonie der Perspektiven. Die Affinität von Prosa zur Schriftlichkeit wäre in diesem Sinne dahingehend auszuweiten, als wohl zunehmend visuelle Bewegungen in Texte und narrative Strukturen in Bildmedien einwandern. Bei keiner der Zeichnungen in Lyophilia handelt es sich um Illustrationen im Paradigma der Bildlichkeit, sondern um visuelle Reflexionen der Darstellungsverfahren dieser Prosa.

\section{Im Sumpf der Prosa}

An einer Stelle in Lyophilia wird dieses Zugleich von Linie, Figur und Bedeutung als das Zugleich von Prosa besonders sichtbar, nämlich dort, wo sich Zladko in einen Grottenolm verwandelt, dessen lateinischer Name nicht umsonst proteus anguinus lautet. Der Olm stürzt sich auf Cottens Zeichnung von einem quergestreiften Strichmuster in ein längsgestreiftes Strichmuster hinein - was Raum und was Leere ist, lässt sich kaum unterscheiden (Cotten 2019, 230). Und während sein Körper bei den Beinen und den Kiemenbüscheln zumindest als solcher erkennbar ist, verliert sich der S-förmige Schwanz (wieder die Schönheitslinie) zu einem Weg durch die Landschaft. Das chimärische Wesen dieser zwischen Figürlichkeit und Abstraktion changierenden Zeichnung entspricht dem chimärischen Wesen des Grottenolms, einer Amphibie, deren Biologie dadurch gekennzeichnet ist, dass sie sowohl an Land als auch im Wasser lebt (Cotten 2019, 230): 
die Peitschenbewegung des Leibes in Zeitlupe an den Posaunenlick, der die Jazzfunk-Nummer Lyophilia in einer anderen Version der Wirklichkeit zum zeitenüberdauernden Hit machte.

"Er wollte nie ein Mensch sein «, sagt Depp.

„Aber das Unfreiwillige stand ihm «, denken die Igore.

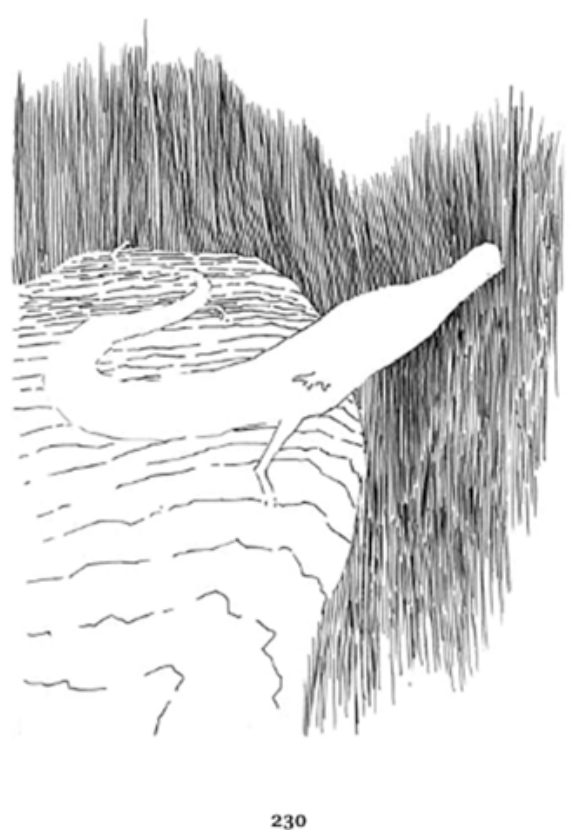

Das Zwischenwesen des Grottenolms auf `Prosa` zu beziehen, liegt weniger fern, als es auf den ersten Blick vielleicht den Anschein hat. Goethe hat in einem Brief an Schiller vom 25. November 1797 "poetische Prosa" folgendermaßen charakterisiert: "Es ist nicht besser, als wenn sich jemand in seinem Park einen trockenen See bestellte und der Gartenkünstler diese Aufgabe dadurch aufzulösen suchte, daß er einen Sumpf anlegte. Diese Mittelgeschlech- 
ter sind nur für Liebhaber und Pfuscher, so wie die Sümpfe für Amphibien. « ${ }^{30}$ >Poetische Prosa ihnen angemessenes Habitat, weil sie selbst - unfähig, sich für etwas zu entscheiden - sowohl das eine wie auch das andere (sein) wollen. Hier wird ex negativo genau das theoretisch formuliert, was bei Cotten sodann als das selbstbewusst Chimärische von Prosa poetologisch auserzählt wird.

Das Sowohl-als-Auch, das suspekt ist, weil es die Einheit der Form auflöst, ist auch das Skandalon einer weiteren Stelle, in der Goethe die verschiedenen Geistes-Epochen, nach Hermanns neusten Mittheilungen darstellt. Seine Gegenwart erscheint als >prosaische Epoche`, in welcher der Verstand alles einnehmen wolle, dadurch aber gerade wieder dem Glauben und dem Mythos Vorschub leiste. So charakterisiert er diese Epoche als eine, in der man »willkürlich Samen und Unkraut zugleich nach allen Seiten« ausstreut, woraus resultiere, dass »Eigenschaften, die sich vorher naturgemäß aus einander entwickelten«, nun »wie streitende Elemente gegen einander [arbeiten] und so ist das Tohu wa Bohu wieder da, aber nicht das erste, befruchtete, gebärende, sondern ein absterbendes, in Verwesung übergehendes, aus dem der Geist Gottes kaum selbst eine ihm würdige Welt abermals erschaffen könnte." (Goethe FA I.20, 245) Auch hier besteht der Effekt von >Prosa darin, dass das, was als einheitliche Form getrennt sein soll, nun wieder zusammenkommt und ambig wird. Es ist gibt keine reinen Arten, sondern alles wird chimärisch miteinander vermischt. Es findet eine Rückwendung in die anfängliche sformlose Leere ‘ des Ursumpfes statt, ${ }^{31}$ jedoch als Regression, die unproduktiv ist - in ihr wird nichts befruchtet und nichts geboren, und selbst Gott könnte daraus nichts mehr schaffen. Auch dies ist also, indem der Fokus auf das Unreine, das Chimärische gelegt wird, eine treffende Beschreibung von >Prosa<, durchaus in Cottens Sinn. Sie korrigiert jedoch das normative Urteil, das mit der mangelnden (Re-)Produktionsfähigkeit dieses Prosa-Sumpfes verbunden ist.

Bevor Zladko selbst zum Grottenolm wird, macht er Bekanntschaft mit seinem Habitat. Ganz zu Beginn der ersten Erzählung von Lyophilia befinden sich Ganja und er in der Postojna-Höhle (die für ihr Grottenolm-Aufkommen bekannt ist) ${ }^{32}$ - und unter den von Touristen bewanderten Holzstegen spielt sich eine prosaische Liebesszene zwischen ihnen ab. Prosa verzichtet hier affirmativ auf den körperlosen, vom Geist bestimmten, vermeintlich übergeordneten, ob-

30 Goethe an Schiller vom 25. November 1797 (FA II.4, 454). Vgl. hierzu auch Barck 2003, 92 sowie Mülder-Bach 2019a, $4 \mathrm{f}$.

31 Die Neue evangelistische Übersetzung des Hebräischen תהו ובהו (১tohu va bohus) lautet ,formlos und leer«, 15. August 2020: https://www.bibleserver.com/NeÜ/1.Mose1 [letzter Zugriff: 15.8.2020].

32 [Postojnska jama:] Geheime Geschichte der Drachenbabys. 
jektiven Standpunkt, den Donna Haraway als »the god trick of seeing everything from nowhere identifiziert hat (Haraway 1988, 581). Unter dem Steg im Schlamm geht es vielmehr um die Einsicht in die eigenen Verstrickungen, die hier in einer Figur, die zugleich ein Wort ist, verdichtet wird (Cotten 2019, 25):

dem Rock und helfe, ihn auszuwringen. Ich habe Angst, dass er zerfällt, aber der Schaum ist mit Polyesterfäden verstärkt, also wird er schlimmstenfalls fadenscheinig. Trotzdem fühlt es sich ziemlich eklig an. Bröckel an den Fingern zwischen dem Schlammwasser. Ich beginne zu wittern, wie ein episch depperter Tag sich aus dem süßen Potential herausfällen könnte, und fluche. Ganja macht mir shhh « und fällt fast um. Ich fange sie auf, und wir sind schon ein Drachen von etwas anderem.

Mit dem Rücken an die Basis des feucht glänzenden Stalagmiten gelehnt, vor dessen oberem Abschnitt die Menschen über uns posieren, braucht sich Ganja nur einen Millimeter bewegen, und ich würde umfallen. Es ist nicht genug Platz, um sich aufzurichten, und auch seitlich können wir uns kaum mehr bewegen als in einer Badewanne, ohne dass irgendein Körperteil unter dem Steg hervorschaute. Wir hocken starr, die Schmerzen der Körperhaltung zählen die Zeit, wir fühlen alle Schauer und Aufregungen beider Körper, unsere jeweilige Wärme, wie sie in die feuchte Kühle loszieht, um sich zu verlieren wie Radiowellen - genau das, als was wir in einem Infrarotsensor zu sehen wären. Wir riechen einander Atem, der, wie Duft, »Ja! Ja! Ja! « ruft. Alles ruft das. Nur unsere Geister sind immer noch zu krustig, zu faul für die weiteren Folgen. Sie legt ihren Kopf mit dem losen Zopf auf meine Schulter, fast hechelnd vor Begehren, im komischen Buchstaben, den wir bilden

\section{互}

und die Fläche meiner Hand geht an ihrer Oberfläche entlang an den Ort, wo Hintern in Bein übergeht, wo die Leggings sich in kleiner Entfernung über ihr Geschlecht spannen, heiß, mir ist, als jauchzte es mir entgegen, ich will fühlen, nehme die Hand aber weg und lege sie an ihre Taille. Darin

Beschrieben wird eine Szene, in der Lust und Schmerz, Kälte und Wärme, Anziehung und Angst vor Abhängigkeit (wenn Ganja sich bewegt, fällt Zladko um) gleichzeitig da sind. Vor allem gilt es, die beiden >streitenden Elemente chelnden Begehrens` und der >zu krustigen Geister` auszutragen. Obwohl ihr 
Atem und die ganze Umgebung »Ja! Ja! Ja!« rufen und damit nach einer romantischen Liebesszene schreien, können sich die beiden zunächst nicht zu irgendwelchen Handlungen entschließen. Sie bilden ineinander - und in ihre jeweils eigenen verkrusteten Vorstellungen - verkeilt »diesen komischen Buchstaben«, den ich zunächst nur als Figur betrachten kann, als Figur zweier ineinander verwickelten Linien bzw. Körper, symmetrisch aufeinander bezogen. Schlage ich die japanische Bedeutung nach, so erfahre ich, dass das Zeichen 互 >gegenseitig`, >gemeinsam ` bedeutet. ${ }^{33}$ Das Zugleich von Prosa ist hier figuriert, einerseits als Gleichzeitigkeit von icon und symbol und andererseits - liest man die Bedeutung poetologisch - als das Miteinander zweier Elemente, die unter Lust und Gewalt zusammenkommen, dabei ein fragiles Muster bildend. Eine Einheit ist dies aber nicht, denn die Vereinigung, die für Goethe notwendig ist, damit das Zeugende von Poesie sich realisieren kann, bleibt hier prekär. Noch als die Figur durch eine plötzliche Bewegung zusammenstürzt und die »Schlammscheu« gebrochen wird (Cotten 2019, 26), bleibt die Annäherung der beiden kompliziert. Sie geschieht eigentlich »durchaus eskapistisch«, d. h. - es sei an das Spazieren/ Schreiben erinnert, das nicht aufgegeben werden kann - als Vermeidung der »komplizierteren Probleme« (Cotten 2018, 39), die dann wieder anfangen, »da es leichter erscheint, zu schmusen, als sich mit der Situation, dass wir irgendwann schlammgetränkt von unter dem Steg hervorkommen müssen, zu beschäftigen« (Cotten 2019, 26). Zladko verkriecht sich zwischen Ganjas Beine: »Ich führe mich mit meiner Zunge in ihren Spalt, kalt heiß, und die Nase wie in Kichern, in ihre Locken.« (Cotten 2019, 26) Doch die Gespaltenheit bleibt auch deswegen bestehen, weil auch dieser Eskapismus wieder eingeholt wird - lässt sich doch die Realität auch inmitten sexueller Handlungen nicht verdrängen: Die »Visionen« der über seinem Kopf paradierenden Sportschuhe lassen ihn »nicht los, egal, was ich mit meiner Zunge mache« (Cotten 2019, 27). Und auch Ganjas Herausforderung besteht darin, dass sie ihren »Geist von der gespaltenen Situation, aufs eigene Kommen und das Kommen von Gefahren gleichermaßen zu achten, in eine einzige Richtung « ziehen soll, was aber zunächst, auch weil sie sich so anstrengt, kaum gelingt. »Doch passiert es - zufällig«, weniger aufgrund von »Meisterschaft« als dank der »Abwesenheit falscher Ideen« (ebd.). >Prosaisch Szene nicht nur, weil romantisch-poetische Versatzstücke durch den Schlamm gezogen werden, sondern weil diese Szene das >Aufspreizende ${ }^{34}$ von Prosa auf allen erdenklichen Ebenen verhandelt: Als Tun und Zögern, Eskapismus

33 [Wikipedia:] 互. https://en.wiktionary.org/wiki/互 [letzter Zugriff: 15.8.2020].

34 Simon 2018, 426: »Prosa ist plurifokal, indem sie ihre Elemente, statt sie auszurichten zum Beispiel auf die Form der Erzählung -, aufspreizt: Der Textraum expandiert in alle Dimensionen gleichzeitig." 
und In-der-Situation-Sein, sowie als zufällige Überlistung des unablässig reflektierenden Selbst durch eine doppelte Ablenkung. Dass Zladko mit seiner Zunge zwischen den Beinen von Ganja verschwindet, und es sich also nicht um eine reproduktive, sondern um eine orale Praktik handelt, bestätigt Goethes Verdacht, wonach Prosa nicht auf Zeugung zielt. Das Chaos ist hier nicht das Befruchtende und Gebärende, es verbleibt im Hin und Her. Dass Entstehen und Vergehen eigentlich dasselbe sind, verdeutlicht denn auch eine andere Stelle in Lyophilia: »Eros wäre dasselbe wie Erosion, Raum-Zeit-Moves in präzisen Dosen an bestimmten Orten, in bestimmte Rhythmen gegossen." (Cotten 2019, 103) Das ständige Zugleich - das Sowohl-als-Auch bzw. nicht ganz das eine und nicht ganz das andere - von >Prosar entzieht sich dem linearen Ablauf von Zeugung, Geburt, Leben und Tod. In Lyophilia erstreckt sich dies auf die Charakterisierung der Figuren, die nie erwachsen werden wollen und sich gleichzeitig immer schon im Sumpf wiederfinden: Sie fürchten sich vor einer abgeschlossenen Form und sind doch schon in den von ihnen unbemerkt selbst geschaffenen Mustern und Texturen gefangen. So versucht der Wecker von Xin - einer weiteren sporadisch auftauchenden Figur aus Lyophilia - ihn mit dem Weck-Ruf zu alarmieren, dass er doch nun mit 35 Jahren endlich erkennen soll, dass »dir niemand anderer aus dem Sumpf deines vermeintlichen Selbst helfen kann als du selbst «, auch wenn - und darin besteht das Problem - »du es aus unerklärlichen Gründen scheinbar nicht vermagst« (Cotten 2019, 12). Erklärbar sind die Gründe schon, denn auch Xin macht die Erfahrung, die Cotten in Was geht formuliert, wonach sich die Seele selbst einlegt wie ein Gemüse: Wie soll man aus den hart erarbeiteten Skills plötzlich herausfinden, sie plötzlich zur Bekämpfung dieser Skills anwenden? Sich am eigenen Schopf aus dem Sumpf zu ziehen, vermochte angeblich nur der Baron von Münchhausen. Der Zirkelschluss oder infinite Regress, der damit verbunden ist, lässt sich in M.C. Eschers Grafik Reptilien (1943), auf die in Lyophilia explizit hingewiesen wird, ${ }^{35}$ zur Anschauung bringen, kann aber nicht gelöst werden.

Fragt man noch einmal nach der Metaphorisierung von Prosa als Möglichkeit einer kulturellen und gesellschaftlichen Diagnose (Mülder-Bach 2019a, 6), so ermöglicht es die >chimärischeく Prosa Cotten, die Gleichzeitigkeit von Fluidität und Verharren, von Veränderungsdrang und Gefangensein in den eigenen Mustern einer Generation in den Blick zu nehmen, der sie - sich selbst eingeschlossen - einen ausgeprägten Besonderheitsdrang attestiert bei gleichzeitiger Sehnsucht nach einem >normalen Leben in einer Gegenwart, die Aleida Assmann als `post-individuelles Zeitalter` bezeichnet hat (Assmann 2006, 213).

35 Cotten 2019, 421: »Aus eurer Erzählung krocht ihr wie H.C. [sic] Eschers erste Amphibien.» 


\section{Leben-Schreiben}

Lyophilia exponiert sich bereits zu Beginn auf vielfältige Weise als `Meta-Literatur ` durch die Meta-Perspektive der Außerirdischen ebenso wie durch die plakativ ausgestellte Autoreflexion (»Wie soll man das erzählen?«). Auch das Aufschieben ebendieses Erzählens sowie die mimetischen Brüche durch die grafischen Irritationen halten die Schwelle zum Text stets präsent. Gleichzeitig hat die Analyse der ersten Seiten gezeigt, dass gerade in der vermeintlichen Position außerhalb die prägenden Muster gebildet werden und sich Systembildung vollzieht. Und umgekehrt hat die Analyse der Sumpf-Szene, mit der die erste `Erzählung`von Lyophilia beginnt, verdeutlicht, dass gerade hier, im und durch das Erzählen, eine implizite Theoretisierung von Prosa in der Anschauung geschieht. Die Irritation eindeutiger Richtungen - sei es vom Diskurs zur Form, wie es Foucault formulierte, sei es von der Figur zur Bedeutung, wie es anhand der Linie sichtbar wurde, oder sei es von Eskapismus und Rückfall in die Situation wie beim Spazieren/Schreiben/Schmusen - erstreckt sich in diesem Text auch auf das Verhältnis von Theorie und Anschauung bzw. Darstellung. `Muster Prosaく schafft als spezifische Dichte in ihren komplexen Texturen eine Meta-Ebene, nur um zu zeigen, dass diese Meta-Perspektive nicht nur von den Mustern produziert, sondern von ihnen auch immer wieder eingeholt und so die Differenz kassiert wird. ${ }^{36}>$ Muster Prosa bringt ihre Bewegungen zur Anschauung und ist so immer Theorie im Vollzug. Zentral für diese Bewegungen ist die Rekursivität, als Faltung wie auch als Hin und Her, in der die Selbstbindung von Prosa sichtbar wird. Was Cottens Texte, sowohl die Poetikvorlesung Was geht als auch Lyophilia, dabei auszeichnet und was für eine >Theorie‘ von Prosa in Hinblick auf die Gegenwart fruchtbar gemacht werden kann, ist, dass Prosa hier nicht nur die Selbstbindung ausstellt, die ihr gegenüber der Lyrik traditionell zugeschrieben wird, sondern auch die Problematik dieser Selbstbindung: In der von Slavoj Žižek oft erzählten Anekdote hat der autoritäre Vater früher dem Kind den Besuch bei der Oma vorgeschrieben, während in der Gegenwart der Besuch vermeintlich freiwillig ist, der Vater dem Kind jedoch das Gefühl

36 Es ist auch bei Cotten durchaus so, dass die Texte tendenziell zur »eigenen Literaturwissenschaft (Metaliteratur) « werden und so das ınarrative Fortkommen« verhindern (Simon 2018, 426), aber es kommt mir darauf an, den Blick auch darauf zu richten, dass sich dabei ebenfalls Muster ausbilden, die diese Meta-Ebene wieder an die Darstellung binden. Auch Simon bemerkt, dass »[a]uf einer tieferen Ebene [...] philosophische und literarisch-fiktionale Architekturen nur noch schwer zu unterscheiden" seien: "Sie teilen als Sprache die grundlegende Paradoxie, dass Referenzbezug bei gleichzeitig elaborierter Selbstreferenz - sei es begriffliche, sei es ästhetische - in sich doppeldeutig und gespalten wird.« (Simon 2013, 285f.) 
gibt: "You must visit your grandma, and you must be glad to do it!« Entzieht sich das Kind dem freiwilligen Besuch, muss es nicht mit der Strafe, sondern dem schlechten Gewissen auskommen (Žižek 1999). Cottens Figuren hatten keine autoritären Väter, sie leiden daran, dass die disziplinarischen Maßnahmen verinnerlicht sind. Und Cottens Prosa, die sich ebenfalls schon längst von normativen Urteilen wie derjenigen des Übervaters Goethe gelöst hat, reflektiert dies als Dilemma der Selbstbindung.

Dieses Dilemma hat Konsequenzen für die Frage »Wie soll man das erzählen?«, die sich immer auch auf die grundsätzliche Frage des Leben-Schreibens bezieht. Ralf Simon hat darauf hingewiesen, dass >Prosa der »hybride Versuch» sei, »auf vor der Form liegende `Lebenskomplexität` mit jenseits der Form liegender sprachlicher Komplexität zu reagieren « und dabei die eigene Unabschließbarkeit zum Thema und zum zentralen Darstellungsverfahren zu machen (Simon 2018, 423). Wo bei Sterne die Uneinholbarkeit der eigenen Autobiografie noch ironisch innerhalb des einen, wenn auch über Jahre hinweg in mehreren Bänden erscheinenden Werks thematisiert wird, ${ }^{37}$ treten Leben und Schreiben bei Jean Paul (hierzu: Pfotenhauer 2020 und 2013), bei Handke oder aber bei Walser in ein Verhältnis gegenseitiger Unabschließbarkeit, das auch jeden neuen Text mit dem vorangehenden verknüpft (Simon 2013, $206 \mathrm{f}$.). Nicht umsonst beschreibt Letztgenannter seine Prosastücke als Teile eines immer im Werden begriffenen und doch nie beendeten immer gleichen Romans, der »als ein mannigfaltig zerschnittenes oder zertrenntes Ich-Buch bezeichnet werden« könnte (Walser 1986, 322). Prosa als Spezialistin für »Langstreckenprobleme« glaubt nicht an die Abschließbarkeit in der Form. Es geht ihr, so Friederike Mayröcker, die Ann Cotten als ihre Wunschenkelin bezeichnet, um das "Weiterspulen oder Weiterspinnen ${ }^{38}$ nicht um das fertige Gewand; sie baut nicht ein einfaches Haus, sondern zieht ein Muster von Hängebrücken zwischen Häuserreihen. Die Werkgrenzen werden aufgeweicht, die exzessive Intertextualität bezieht sich nicht nur auf andere Autor`innen, sondern entsteht innerhalb eines Werks, bei dem das nächste Buch das vorangehende weiterschreibt. `Muster-Prosa verwirrt nicht nur, wie

37 Sterne 2010, 331: »Ich bin in diesem Monat um ein ganzes Jahr älter, als ich heute vor zwölf Monaten war; und da ich in dieser Zeit, wie Sie bemerken werden, fast bis in die Mitte meines dritten Bandes gekommen bin - und es doch nicht weiter gebracht habe als bis zu meinem ersten Lebenstage, so folgt daraus klar, daß ich jetzt 364 Tage mehr von meiner Lebensbeschreibung zu schreiben habe, als da ich anfing; so daß ich statt wie jeder andere Schriftsteller mit dem, was ich bis jetzt daran getan, weiter vorwärts zu kommen - ich im Gegenteil um ebensoviele Bände zurückgekommen bin.«

38 So Mayröcker in einem Gespräch mit Dieter Sperl, zit. n. Kunz 2004, 12. 
Schklowski konstatiert, den geraden Faden der Autobiografie, sondern auch die Richtungen zwischen Leben und Kunst, Kunst und Leben. ${ }^{39}$

Zwar inszeniert Tristam Shandy ein Erzählen ab ovo, aber statt dem Erzähler und Protagonisten schnell zu einer guten Geburt zu verhelfen, sorgt Prosa für ständigen Aufschub - ein Aufschub, der bei Cotten widerhallt als Nicht-Erwachsenwerden-Wollen und der sein Komplement im Nicht-Aufhören-Können und in den sich daraus ergebenden spezifischen »Langstreckenprobleme[n] « hat.

Das Dilemma, angesichts vermeintlich unendlicher Möglichkeiten nur die Selbstbindung zu haben, die einen zwingt, Muster auszubilden, in denen man sich dann wieder verheddert, ist nicht zu lösen. Es ist nur ein spielerischer Umgang damit möglich. In Was geht verschiebt sich so der Fokus vom anfänglichen Besonderheitsdrang der Spaziergängerin mit ihren Digressionen auf das »interessante Mittelfeld «, wo die Leute einfach »gut zusammenarbeiten « (Cotten 2018, 133f.). Dabei muss die Exzentrik nicht ganz aufgegeben werden (das wäre dann doch unmöglich), so die vergleichbare Einsicht, die auch Zladko in Lyophilia formuliert: »Wie beim Handstand geht es beim Leben darum zu schauen, wie lang man es schafft. Ohne Ausgang, ohne Ziel, ohne Ende. Immer passiert irgendetwas, es wird hell, man muss zu irgendwelchen Terminen, duschen. Kooperativ. Schön, normal halt, Arbeit.« (Cotten 2019, 107) Die Frage ist nicht: Gibt es in der Kontingenz des Lebens ein Muster, sondern: Wie bilden sich aus der Reihe von Ungewolltem und Zufälligem Muster heraus? Und wie ist damit umzugehen? Bei Prosa wie im Leben gilt es anzuerkennen, dass man zwangsläufig Muster ausbildet, man im Schlamm versumpft und die Seele vergurkt. Aber hier ist nicht mehr die Kritik am chimärischen Zeitalter hörbar, die über die eigene Nachlässigkeit klagt, und auch keine emphatische Rede einer ızukünftigen Art`. Vielmehr zeichnet sich ein pragmatisch-ironischer Blick ab: Zwar kommt man mit Artistik auch nicht weiter (man wird vielleicht besser, balanciert aber am gleichen Ort); man kann aber nur immer wieder versuchen und »schauen, wie lang man es schafft«. Wenn man nicht damit beschäftigt ist, sich zu einer ganz speziellen Form zu bilden, dann passiert dort, wo man nicht hinschaut, doch etwas - es passiert: Leben. Und es passiert: Prosa.

39 Vgl. auch Simon 2018, 423: "Das Leben ist immer schon ausgedeutet und muss immer noch von Anfang an gedacht werden; immer ist alles schon als Wiederholung gelesen und doch muss es als Anfang inszeniert werden. Prosa überholt den traditionellen Ästhetizismus, nach dem das Leben die Kunst imitiere, durch eine doppelte Rekursionsfigur, die letztlich auf die rätselhafte und ins Humoristische mündende Identifikation von Leben und Konstruktion hinausläuft.» 


\section{Literaturverzeichnis}

Agamben, Giorgio: Idee der Prosa. In: ders.: Idee der Prosa. Frankfurt a. M. 2003, 21-24. Albes, Claudia: Der Spaziergang als Erzählmodell. Studien zu Jean-Jacques Rousseau, Adalbert Stifter, Robert Walser und Thomas Bernhard. Tübingen 1999.

Andres, Susanne: Robert Walsers arabeskes Schreiben. Göttingen 1997.

Assmann, Aleida: Generationsidentitäten und Vorteilsstrukturen in der neuen deutschen

Erinnerungsliteratur. Wien 2006.

Assmann, Aleida: Im Dickicht der Zeichen. Berlin 2015.

Barck, Karlheinz: Prosaisch-poetisch. In: Ästhetische Grundbegriffe. Historisches Wörterbuch in sieben Bänden. Bd. 5: Postmoderne - Synästhesie. Hrsg. v. Karlheinz Barck et al.

Stuttgart, Weimar 2003, 87-112.

Baumgärtel, Tilman: Schleifen. Zur Geschichte und Ästhetik des Loops. Berlin 2016. Bedenk, Jochen: Verwicklungen. William Hogarth und die deutsche Literatur des

18. Jahrhunderts (Lessing, Herder, Schiller, Jean Paul). Würzburg 2004.

Benjamin, Walter: Robert Walser. In: ders.: Gesammelte Schriften. Bd. II/1. Hrsg. v. Rolf

Tiedemann und Hermann Schweppenhäuser. Frankfurt a. M. 1991, 324-328.

Boehm, Gottfried, Gabriele Brandstetter und Achatz von Müller (Hg.): Figur und Figuration.

Studien zu Wahrnehmung und Wissen. Paderborn 2007.

Bonnefoit, Régine: Der »Spaziergang des Auges« im Bilde. Reflexionen zur Wahrnehmung von

Kunstwerken bei William Hogarth, Adolf von Hildebrand und Paul Klee. In: kritische

berichte - Zeitschrift für Kunst- und Kulturwissenschaften 32.4 (2004), 6-18.

Bonnefoit, Régine: Die Linientheorien von Paul Klee. Petersberg 2009.

Campe, Rüdiger: Das Problem der Prosa und die Form des Romans. Überlegungen zu Friedrich

Schlegels Theorie und Praxis um 1800. In: Die Farben der Prosa. Hrsg. v. Eva Eßlinger, Heide Volkening und Cornelia Zumbusch. Freiburg i. Brsg., Berlin, Wien 2016, 45-63.

Cixous, Hélène: Gespräch mit dem Esel. Blind schreiben. Versehen mit zwei Supplementen.

Hrsg. v. Esther Hutfless und Elisabeth Schäfer. Wien 2018.

Cotten, Ann: Was geht. Wien 2018.

Cotten, Ann: Lyophilia. Berlin 2019.

Driesen, Christian: Über Kritzeln. Graphismen zwischen Schrift, Bild, Text und Zeichen. Zürich 2012.

Efimova, Svetlana und Michael Gamper (Hg.): Prosa. Geschichte, Poetik, Theorie. Berlin, Boston 2021.

Foucault, Michel: Dies ist keine Pfeife. Mit zwei Briefen und vier Zeichnungen von René Magritte. Aus dem Franz übers. u. mit einem Nachw. v. Walter Seitter. München, Wien 1997.

Gamper, Michael: Rhythmus - eine Organisationsform der Prosa. In: Prosa. Geschichte, Poetik, Theorie. Hrsg. v. Svetlana Efimova und Michael Gamper. Berlin, Boston 2021, 35-49.

Goethe, Johann Wolfgang: Sämtliche Werke, Briefe, Tagebücher und Gespräche. 40 Bde. Hrsg. v. Henrik Birus et al. Frankfurt a. M. 1985-1999 [= FA].

Graevenitz, Gerhart von: Das Ornament des Blicks. Über die Grundlagen des neuzeitlichen Sehens, die Poetik der Arabeske und Goethes West-östlichen Divan. Stuttgart, Weimar 1994. Graf, Daniel: Buchstäblich verspielt. In: Republik (18.1.2019). Das digitale Magazin für Politik, Wirtschaft, Gesellschaft und Kultur. 15. August 2020: https://www.republik.ch/2019/01/ 18/buchstaeblich-verspielt. 
Hahn, Daniela, Ansgar Mohnkern und Rolf Parr (Hg.): Kulturelle Anatomien: Gehen. Heidelberg 2017.

Handke, Peter: Am Felsfenster morgens (und andere Ortszeiten 1982-1987). Salzburg, Wien 1998.

Haraway, Donna: Situated Knowledges. The Science Question in Feminism and the Privilege of Partial Perspective. In: Feminist Studies 14.3 (1988), 575-599.

Hogarth, William: Analyse der Schönheit. Aus dem Eng. v. Jörg Heiniger. Dresden, Basel 1995.

Honold, Alexander: Der Erd-Erzähler. Peter Handkes Prosa der Orte, Räume und Landschaften. Stuttgart 2017.

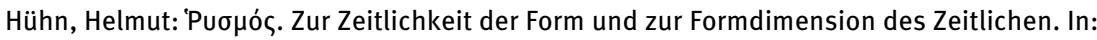
Denkfigur Rhythmus. Probleme und Potenziale des Rhythmusbegriffs in den Künsten. Hrsg. v. Boris Roman Gibhardt. Hannover 2020, 73-89.

Jean Paul: Vorschule der Ästhetik. In: ders.: Werke. Bd. 5. Hrsg. v. Norbert Miller. München 1963.

Kain, Thomas et al. (Hrsg.): Paul Klee in Jena 1924. Die Ausstellung - der Vortrag. Ausst.-Kat. Stadtmuseum Göhre. 2 Bde. Jena 1999.

Keller, Claudia: Zeitfiguren des Epischen. Peter Handkes Die Obstdiebin als eine Theorie des Erzählens im Erzählen. In: Zeitschrift für deutsche Philologie 139.4 (2020a), 591-619.

Keller, Claudia: `Dier` nervt. Ann Cotten liest Peter Handke. In: Weimarer Beiträge 4 (2020b), 544-565.

Klee, Paul: Pädagogisches Skizzenbuch. München 1925.

Kracauer, Siegfried: Theorie des Films. Die Errettung der äußeren Wirklichkeit. Mit einem Anhang »Marseiller Entwurf« zu einer Theorie des Films. Hrsg. v. Inka Mülder-Bach. Unter Mitarb. v. Sabine Biebl. Frankfurt a. M. 2005.

Krämer, Sybille und Horst Bredekamp: Bild, Schrift, Zahl. München 2003.

Krämer, Sybille und Eva Cancik-Kirschbaum (Hg.): Schriftbildlichkeit. Wahrnehmbarkeit, Materialität und Operativität von Notationen. Berlin 2012.

Kunz, Edith Anna: Verwandlungen. Zur Poetologie des Übergangs in der späten Prosa Friederike Mayröckers. Göttingen 2004.

Lüdemann, Susanne: Ungebundene Rede. Prosa und die Frage der Form. In: Prosa schreiben. Literatur - Geschichte - Recht. Hrsg. v. Inka Mülder-Bach, Jens Kersten und Martin Zimmermann. München 2019, 309-324.

Lutes, Jason: Berlin. Montréal 2018.

Malewitsch, Kasimir: Die gegenstandslose Welt. München 1927.

Mersmann, Birgit: Schriftikonik. Bildphänomene der Schrift in kultur- und medienkomparativer Perspektive. München 2015.

Mülder-Bach, Inka, Jens Kersten und Martin Zimmermann (Hg.): Prosa schreiben. Literatur Geschichte - Recht. München 2019.

Mülder-Bach, Inka: Einleitung. In: Prosa schreiben. Literatur - Geschichte - Recht. Hrsg. v. dies., Jens Kersten und Martin Zimmermann. München 2019a, 1-14.

Mülder-Bach, Inka: Die Prosa der Gesellschaft. Literarische Form und soziale Bindung in Goethes Unterhaltungen deutscher Ausgewanderten. In: Prosa schreiben. Literatur Geschichte - Recht. Hrsg. v. Inka Mülder-Bach, Jens Kersten und Martin Zimmermann. München 2019b, 225-248.

Naumann, Barbara: Die »kolossale Kritzelei«, der »borghesische Fechter« und andere Versuche. In: Der grüne Heinrich. Gottfried Kellers Lebensbuch - neu gelesen. Hrsg. v. Wolfram Groddeck. Zürich 2009, 159-200. 
Pfotenhauer, Helmut: Jean Paul. Das Leben als Schreiben. München 2013.

Pfotenhauer, Helmut: "Das wahre Leben ist die Literatur «. Konzepte radikaler Autorschaft von Jean Paul bis Robert Walser. Würzburg 2020.

[Postojnska jama:] Geheime Geschichten der Drachenbabys. 15. August 2020: https://www. postojnska-jama.eu/de/geschichten-der-wunderwelt/geheime-geschichten-der-drachen babys/.

Rosenblum, Robert: Transformations in late eighteenth century art. Princeton 1967.

Scheffler, Kirsten: Mikropoetik. Robert Walsers Bieler Prosa. Spuren in ein »Bleistiftgebiet» avant la lettre. Bielefeld 2010.

Schklowski, Viktor: Ornamentale Prosa. In: Die Erweckung des Wortes. Essays der russischen formalen Schule. Hrsg. v. Fritz Mierau. Leipzig 1987, 88-111.

Schlegel, Friedrich: Geschichte der Poesie der Griechen und Römer. Bd. 1.1. Berlin 1798.

Simon, Ralf: Die Idee der Prosa. Zur Ästhetikgeschichte von Baumgarten bis Hegel mit einem Schwerpunkt bei Jean Paul. München 2013.

Simon, Ralf: Vor und nach der Form. Zur Temporalität des ästhetischen Formprozesses. In: Zeit der Form - Formen der Zeit. Hrsg. v. Michael Gamper, Eva Geulen, Johannes Grave, Andreas Langenohl, Ralf Simon und Sabine Zubarik. Hannover 2016, 63-82.

Simon, Ralf: Theorie der Prosa. In: Grundthemen der Literaturwissenschaft. Poetik und Poetizität. Hrsg. v. Ralf Simon. Berlin 2018, 415-429.

Sterne, Laurence: Leben und Meinungen von Tristram Shandy, Gentleman. Aus dem Engl. übers. v. Otto Weith. Nachwort v. Erwin Wolff. Stuttgart 2010.

Walser, Robert: Selected Stories. With a foreword by Susan Sontag, transl. by Christopher Middleton. Manchester 1982.

Walser, Robert: Eine Art Erzählung. In: ders.: Sämtliche Werke in Einzelausgaben. Bd. 20: Für die Katz. Prosa aus der Berner Zeit 1928-1933. Hrsg. v. Jochen Greven. Zürich, Frankfurt a. M. 1986, 322.

[Wikipedia:] 互.15. August 2020: https://en.wiktionary.org/wiki/互.

Žižek, Slavoj: ıYou May! - The Postmodern Super-Ego. In: London Review of Books 21.61999. 16. August 2020: https://www.lrb.co.uk/the-paper/v21/n06/slavoj-zizek/you-may!.

Zollinger, Albin: Robert Walsers Roman >Der Gehülfes. In: Über Robert Walser. Bd. 1. Hrsg. v. Katharina Kerr. Frankfurt a. M. 1978, 132-138. 